\title{
Birational Geometry of the Space of Complete Quadrics
}

\author{
BY \\ CÉSAR ADRIÁN LOZANO HUERTA
}

\begin{abstract}
THESIS
Submitted in partial fulfillment of the requirements

for the degree of Doctor of Philosophy in Mathematics in the Graduate College of the University of Illinois at Chicago, 2014
\end{abstract}

\author{
Chicago, Illinois
}

Doctoral Committee:

Professor Izzet Coskun, Chair

Professor Tommaso de Fernex, University of Utah

Professor Lawrence Ein

Professor Mihnea Popa

Research Assistant Professor Jack Huizenga 
Copyright by

César Adrián Lozano Huerta

2014 
A las mujeres de mi vida:

Amalia, Teresita de Jesús, Delfa, Alejandra y Rita. 


\section{ACKNOWLEDGMENTS}

This work has been possible thanks to the generous financial support of the Mexican National Council of Science and Technology (CONACyT) and The University of Illinois at Chicago.

I have been extremely privileged to be a student of Izzet Coskun. He has been an inspirational figure throughout my graduate studies and will surely remain being so. He is responsible I am an algebraic geometer; to him my deepest gratitude.

This work and my understanding of mathematics have benefited a great deal from conversation with Dawei Chen, Lawrence Ein, Jack Huizenga, Mihnea Popa, Christian Schnell, Kevin Tucker and Francesco Cavazzani.

Thanks to my family Rita, Adrián, Tomás, Enrique, Fernando for all your support and love.

Thanks to all my dear friends who made of my stay in Chicago a truly wonderful lifetime experience. In particular, Chris Gomez, Alex Stathis, Tim Ryan, Alex Austin, Patricio Gallardo, Jonathan Schneider, Jonah Gaster, Andrew Lawrie, Chih-Chi Chou, David Chen, Elisa Wan, Richard Abdelkerim, Dan Ingebretson, Ellie Dannenberg, Sam Ziegler.

Thanks to Renzo Cavalieri, my advisor in Mexico City Ernesto Lupercio and Emigdio Salazar. I would never have become a mathematician without them. 


\section{AGRADECIMIENTOS}

Este trabajo ha sido posible gracias al generoso financiamiento del Consejo Nacional de Ciencia y Tecnología (CONACyT) y la Universidad de Illinois en Chicago.

He sido extremadamente afortunado de ser estudiante de Izzet Coskun. Él ha sido una

figura inspiradora a lo largo de mis estudios de posgrado y seguramente lo segirá siendo en el futuro; par él mi más profunda gratitud.

Este trabajo y mi entendimiento de las matemáticas se han beneficiado en gran medida de conversaciones con Dawei Chen, Lawrence Ein, Jack Huizenga, Mihnea Popa, Christian Schnell, Kevin Tucker y Francesco Cavazzani.

Mil gracias a mi familia Rita, Adrián, Tomás, Enrique, Fernando por todo el amor y apoyo.

Mil gracias a todos mis queridos amigos que hicieron de mi estancia en Chicago una experiencia de vida maravillosa. En especial Chris Gomez, Alex Sthatis, Tim Ryan, Alex Austin, Patricio Gallardo, Jonathan Schneider, Jonah Gaster, Andrew Lawrie, Chih-Chi Chou, David Chen, Elisa Wan, Richard Abdelkerim, Dan Ingebretson, Ellie Dannenberg, Sam Ziegler.

Gracias a Renzo Cavalieri, mi asesor en la Ciudad de México Ernesto Lupercio y Emigdio Salazar. Sin ellos, jamás me hubera convertido en matemático. 


\section{TABLE OF CONTENTS}

CHAPTER

PAGE

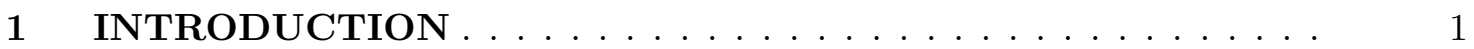

2 CYCLES ON THE SPACE OF COMPLETE QUADRICS . . . . 8

$2.1 \quad$ Cycles of codimension one . . . . . . . . . 8

2.2 Cycles of codimension two on the space of complete conics . . 12

3 MORI'S PROGRAM FOR COMPLETE $(N-1)$-QUADRICS . . 19

$3.1 \quad$ Mori's program in arbitrary dimensions. . . . . . . . . . . 19

$3.2 \quad$ Mori's program for complete quadric surfaces . . . . . . . . . 23

3.3 Stable base locus decomposition . . . . . . . . . . . 28

$3.4 \quad$ Birational models of complete quadric surfaces . . . . . . . . 34

3.5 Proof of Theorem B . . . . . . . . . . . . . 41

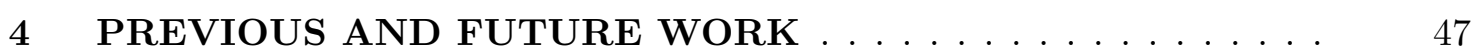

CITED LITERATURE $\ldots \ldots \ldots \ldots \ldots \ldots \ldots$ 


\section{LIST OF ABBREVIATIONS}

$\begin{array}{ll}X & \text { Space of complete }(n-1) \text {-quadrics } \\ \text { MMP } & \text { Minimal Model Program } \\ X(D) & \text { Model of } X \text { induced by a divisor } D \\ \operatorname{Eff}(X) & \text { Cone of effective divisors of } X \\ \operatorname{Nef}(X) & \text { Flip of } X \text { over Chow }{ }_{2}(1, X) \\ X^{+} & \text {Second order Chow variety } \\ \text { Chow }{ }_{2}(1, X) & \text { Hilbert scheme of conics on } \mathbb{G}(1,3) \\ \text { Hilb } & \text { Mori cone of curves on } X\end{array}$




\section{SUMMARY}

Let $X$ be the moduli space of complete $(n-1)$-quadrics. In this thesis, we study the birational geometry of $X$ using tools from the minimal model program (MMP). In Chapter 1, we recall the definition of the space $X$ and summarize our main results in Theorems A, B and C.

In Chapter 2, we examine the codimension-one cycles of the space $X$, and exhibit generators for $\operatorname{Eff}(X)$ and $\operatorname{Nef}(X)$ (Theorem A), the cone of effective divisors and the cone of nef divisors, respectively. This result, in particular, allows us to conclude the space $X$ is a Mori dream space.

In Chapter 3, we study the following question: when does a model of $X$, defined as $X(D):=$ $\operatorname{Proj}\left(\bigoplus_{m \geq 0} H^{0}(X, m D)\right)$, have a moduli interpretation? We describe such an interpretation for the models $X\left(H_{k}\right)$ (Theorem B), where $H_{k}$ is any generator of the nef cone $\operatorname{Nef}(X)$. In the case of complete quadric surfaces there are 11 birational models $X(D)$ (Theorem B), where $D$ is a divisor in the movable cone $\operatorname{Mov}(X)$, and among which we find a moduli interpretation for seven of them.

Chapter 4 outlines the relation of this work with that of Semple (1), (2) as well as future directions of research. 


\section{CHAPTER 1}

\section{INTRODUCTION}

This thesis studies the birational geometry of the space of complete quadrics.

The moduli spaces of complete quadrics are compactifications of the space of smooth quadric hypersurfaces in $\mathbb{P}^{n}$. These spaces were introduced by Schubert (3) and studied by many prominent mathematicians of the 19th century such as Schubert, Chasles and Hirst. Complete quadrics were instrumental in the development of enumerative geometry and intersection theory carried out by Kleiman, Laksov and others (4). Moreover, these spaces are important examples of "wonderful varieties" which are special compactifications of symmetric varieties defined and studied in representation-theoretic terms (5). This dissertation aims to exploit all these different perspectives of these classical spaces and draw conclusions that relate their moduli theory and their birational geometry.

To study the birational geometry of the space of complete quadrics we will use the Minimal Model Program (MMP). Specifically, we want to understand the birational geometry of the space of complete $(n-1)$-quadrics $X$ by examining all the morphisms (possibly rational) from $X$ to another projective algebraic variety. We will do so, following the fundamental work of Mori, by examining the geometry of certain convex cones called the cone of numerically effective divisors, nef cone $\operatorname{Nef}(X)$ (see $1.1(6)$ ), and the cone of effective divisors, $\operatorname{Eff}(X)$, (see $1.1(6))$ both contained in the Neron-Severi group $N^{1}(X)$, which is the space of divisors up to 
numerical equivalence. Our first result, Theorem A, describes the geometry of these two such cones. Before stating such a theorem, let us introduce precisely the objects we will study.

We recall the definition of the main object of study of this dissertation. We work over the field of complex numbers throughout. Let $Q \subset \mathbb{P}^{n}=\mathbb{P}(V)$ be a smooth quadric hypersurface. It defines a symmetric linear map $Q: V \rightarrow V^{*}$, which induces a symmetric linear map $\Lambda^{k} Q$ : $\Lambda^{k} V \rightarrow \Lambda^{k} V^{*}$ for any $1 \leq k \leq n$. Hence, $\Lambda^{k} Q$ can be thought of as a quadric, i.e., an element in $S^{2}\left(\Lambda^{k} V\right)$. If $Q$ is smooth, then the association $Q \mapsto \Lambda^{k} Q$ is injective up to multiplication by scalars. Consequently, we get an embedding of $X^{\circ}$, the family of smooth quadric hypersurfaces in $\mathbb{P}(V)$, into the space $W=\mathbb{P}\left(S^{2}(V)\right) \times \mathbb{P}\left(S^{2}\left(\Lambda^{2} V\right)\right) \times \ldots \times \mathbb{P}\left(S^{2}\left(\Lambda^{n} V\right)\right)$ via the map

$$
\rho: Q \mapsto\left(Q, \Lambda^{2} Q, \ldots, \Lambda^{n} Q\right)
$$

Definition 1.1. Let the space of complete $(n-1)$-quadrics $X$ be the closure $\overline{\rho\left(X^{\circ}\right)} \subset W$.

Let us introduce the generators of the nef cone $\operatorname{Nef}(X)$, whose first mention can already be found in Schubert (3).

Definition 1.2. Let $X$ denote the space of complete $(n-1)$-quadrics. Let $H_{i} \subset X$ be the closure in $X$ of the subvariety parametrizing smooth quadric hypersurfaces in $\mathbb{P}^{n}$ which are tangent to a fixed linear subspace of dimension $i-1$.

Let us now introduce the generators of the effective cone $\operatorname{Eff}(X)$. The Lie group $S L_{n+1} \mathbb{C}$ acts on $X$ with the following properties: there is an open dense $S L_{n+1} \mathbb{C}$-orbit in $X$ which is isomorphic to $X^{\circ}:=S L_{n+1} / N$, where $N$ is the subgroup fixed by the involution $\sigma(A)={ }^{t} A^{-1}$. 
Outside such an open dense subset, $X \backslash X^{\circ}$ is the union $\bigcup_{i}^{l} E_{i}$ of smooth boundary divisors $E_{i}$ with transversal intersection, where $l$ denotes the dimension of the maximal anisotropic torus of $S L_{n+1} / N$.

Theorem A. Let $X$ be the space of complete $(n-1)$-quadrics. The cone of effective divisors on $X$ is generated by boundary divisors $\operatorname{Eff}(X)=\left\langle E_{1}, \ldots, E_{n}\right\rangle$. Furthermore, the nef cone is generated by $\operatorname{Nef}(X)=\left\langle H_{1}, \ldots, H_{n}\right\rangle$.

This description of the nef cone $\operatorname{Nef}(X)$ allows us to conclude that the space $X$ is a Fano variety. As a consequence of being a Fano variety, following (7), the space $X$ is a Mori dream space. The notion of Mori dream space was introduced by Keel and $\mathrm{Hu}$ in (8) and it gives name to those $\mathbb{Q}$-factorial algebraic varieties for which $N^{1}(X) \otimes \mathbb{Q}=\operatorname{Pic}(X) \otimes \mathbb{Q}$ and whose Cox ring is finitely generated. One of the main properties of a Mori dream space is that the cone of effective divisors $\operatorname{Eff}(X)$ and the cone of nef divisors $\operatorname{Nef}(X)$ are rational polyhedral. Furthermore, the cone of effective divisors can be decomposed into a finite number of the socalled Mori chambers which are rational polyhedral convex subcones. The relevance of these chambers is explained next.

Suppose our goal is to understand all the morphisms from a $\mathbb{Q}$-factorial variety $X$ to another projective variety $Y$, then in order to avoid redundancies we observe that for each line bundle $\mathcal{L} \in \operatorname{Pic}(X) \otimes \mathbb{Q}$ over $X$, the linear system induces a rational map $\phi_{m}: X \rightarrow \mathbb{P}\left(H^{0}\left(X, \mathcal{L}^{\otimes m}\right)\right)$ which for sufficiently large and divisible $m$ gives rise to the "Iitaka fibration" (see 2.1 in (6)). Two line bundles $\mathcal{L}_{1}$ and $\mathcal{L}_{2}$ are called Mori equivalent if they give rise to equivalent Iitaka 
fibrations $\phi_{\mathcal{L}_{1}}$ and $\phi_{\mathcal{L}_{2}}$. In a Mori dream space $X$ we have that $N^{1}(X) \otimes \mathbb{Q}=\operatorname{Pic}(X) \otimes \mathbb{Q}$, and the Mori chambers are the closures of the equivalence classes of Mori equivalent line bundles.

As a consequence of the discussion above, the moduli space $X$ will have finitely many equivalence classes of maps $\phi_{D}: X \rightarrow Y$, where $D \in \operatorname{Eff}(X)$. The closure of the image of a map $\phi_{D}$ will be called a model of $X$. More precisely,

Definition 1.1. Let $X$ be a smooth projective variety and let $D$ be a Cartier divisor on $X$. Assume that the algebra $R(X, D):=\bigoplus_{m \geq 0} H^{0}(X, m D)$ is finitely generated. We define the model of $X$ induced by $D$,

$$
X(D):=\operatorname{Proj} R(X, D) .
$$

Those divisors $D$ for which the map $\phi_{D}$ is an isomorphism in codimension one are called small modifications of $X$ and are of special importance: they give rise to divisorial contractions and flips of $X$ (definition 3.4). Such divisors are called movable and they form the so-called movable cone $\operatorname{Mov}(X)$, to be defined in 3.2.

In sum, the fact that $X$ is a Mori dream space means we can run the MMP on $X$ and expect finitely many Mori equivalent models $X(D)$ as the divisor $D$ varies in $\operatorname{Eff}(X)$. However, Mori chambers, which control such models $X(D)$, are very difficult to compute. So, in order to describe the Mori chamber decomposition we use the fact that the Mori chambers can be identified by looking at the stable base locus of the respective divisors. This relation among Mori chambers and the stable base locus decomposition has been studied in $(9 ; 10)$. Let $X$ be the moduli space of complete quadric surfaces. In Proposition 3.3 we determine the stable base 
locus decomposition of the cone of effective divisors $\operatorname{Eff}(X)$. Once we have such a decomposition it is natural to ask, can we explicitly describe the small modifications of $X$ ? Are such small modifications compactifications of the family of smooth quadric hypersurfaces? Are the small modifications of $X$ derived from classical constructions? We provide a constructive answer.

Theorem B. The cone of effective divisors of the space of complete quadric surfaces has eight Mori chambers. Furthermore, we find a moduli interpretation for many of the birational models induced by divisors in the movable cone $\overline{\operatorname{Mov}}(X)$.

This result contains one of the main goals of this dissertation: Theorem B makes a connection between the birational geometry of the space $X$ and its moduli theory. Remarkably, models obtained by running the MMP on a moduli space often have themselves a moduli interpretation. A priori, there is no reason for this to be the case. However, Hassett and Keel first exhibited this phenomenon in the context of the Deligne-Mumford compactification of the moduli space of Riemann surfaces $\overline{\mathcal{M}}_{g}(11),(12),(13)$. This has been the leading example, and it drives a good deal of the research on the birational geometry of moduli spaces.

Another example of this phenomenon, which is closely related to complete quadrics and will be relevant to this dissertation, is the following. Let $\bar{M}=\bar{M}_{0,0}(\mathbb{G}(1,3), 2)$ denote the Kontsevich moduli space of stable maps of degree two into the Grassmannian. Chen and Coskun run the MMP on this moduli space and describe its small modifications (14). Moreover, they found that such modifications of $\bar{M}$ have a moduli structure. For example, the Hilbert scheme 
Hilb $=$ Hilb $^{2 x+1}(\mathbb{G}(1,3))$ of conics in the Grassmannian is the flip of $\bar{M}_{0,0}(\mathbb{G}(1,3), 2)$ over $\mathcal{C}$ the Chow variety of conics in the Grassmannian. Schematically,

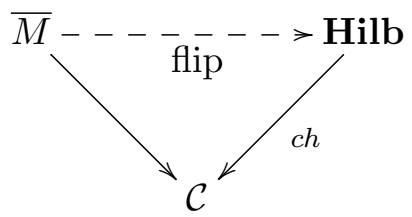

where ch denotes the Hilbert-Chow morphism.

Theorem B says that the same remarkable phenomenon, first found by Hassett and Keel, holds true for the models of the moduli space of complete quadric surfaces. Finding a moduli interpretation for the models $X(D)$ will be called Mori's program for complete $(n-1)$-quadrics throughout the dissertation.

Observe that all of the models $X(D)$ in Theorem B are derived from classical constructions. For example, we get the model $X\left(H_{2}\right)=$ Chow $_{2}(1, X)$ by associating a quadric line-complex to a smooth quadric (see section 3.4). This is a particular case of the following construction.

The second order Chow variety $\mathbf{C h o w}_{2}(k, X)$ parametrizes tangent $k$-planes to complete $(n-1)$-quadrics. In other words, if $Q \subset \mathbb{P}(V)$ is a smooth quadric, then the tangent $k$-planes to $Q$ are parametrized by the Chow form $C F_{Q} \subset \mathbb{G}(k, n)$, which is a degree 2 divisor. Thus $[C F]$ is an element in $\left|\mathcal{O}_{\mathbb{G}}(2)\right| \subset \mathbb{P}\left(S^{2}\left(\Lambda^{k} V\right)\right)$. The association $Q \mapsto C F_{Q}$, induces a birational morphism

$$
\rho_{k}: X \rightarrow \mathbb{P}\left(S^{2}\left(\Lambda^{k} V\right)\right)
$$


We define the second order Chow variety $\mathbf{C h o w}_{2}(k-1, X)$ as the image of $\rho_{k}$.

Theorem C. Let $X$ be the space of complete $(n-1)$-quadrics. The birational model $X\left(H_{k}\right)$ induced by any generator of the nef cone $\operatorname{Nef}(X)$ is isomorphic to $\mathbf{C h o w}_{2}^{\nu}(k-1, X)$ the normalization of the second order Chow variety.

We now want to look at the space of complete quadrics from the perspective of representation theory. For a detailed treatment on wonderful varieties we refer the reader to (5).

It is known that the space of complete $(n-1)$-quadrics $X$ is isomorphic to the "wonderful compactification" of the Lie quotient $X^{\circ}=S L(n+1, \mathbb{C}) / \tilde{S O}(n+1)$. This compactification is constructed by finding a suitable irreducible representation of $S L(n+1, \mathbb{C})$ and a vector $v$, whose stabilizer is the subgroup $\tilde{S O}(n+1)$. This construction recovers the model $X(D)$ where $D$ is an ample divisor.

A natural question is whether the birational models $X(D)$ studied in this thesis are also compactifications for the same quotient $X^{\circ}$. This is the case for all the instances in this dissertation. Hence, it motivates the following question: do all the models $X(D)$ arise in a similar representation-theoretic fashion? In other words, if $D \subset X$ is a movable divisor (Definition 3.2), what are the representations of $S L(n+1, \mathbb{C})$ which give rise to compactifications of $X^{\circ}$ that are isomorphic to the model $X(D)$ ? In Chapter 4, we comment on these ideas in more detail and explain how they lead to future directions of research. 


\section{CHAPTER 2}

\section{CYCLES ON THE SPACE OF COMPLETE QUADRICS}

In this chapter we prove Theorem A. This result exhibits generators for $\operatorname{Eff}(X)$ and $\operatorname{Nef}(X)$, the cone of effective divisors and nef divisors, respectively. The strategy used in the proof of this theorem can be used to analyze higher codimension cycles. In section 2.2 we compute $\mathrm{Eff}^{2}(X)$, the cone of codimension-two effective cycles on the space of complete conics.

\subsection{Cycles of codimension one}

The following result yields an alternative description for the space of complete $(n-1)$ quadrics $X$. Let $\mathbb{P}^{N}$, where $N=\left(\begin{array}{c}n+2 \\ 2\end{array}\right)-1$, be the space parametrizing quadric hypersurfaces in $\mathbb{P}^{n}$. We can stratify $\mathbb{P}^{N}$ in terms of the rank of the quadrics hypersurfaces,

$$
\Phi_{1} \subset \cdots \subset \Phi_{n-1} \subset \Phi_{n} \subset \mathbb{P}^{N}
$$

where $\Phi_{i}$ denotes the locus of quadrics of rank at most $i$. The space of complete quadrics is obtained as a sequence of blowups of $\mathbb{P}^{N}$ along all the $\Phi_{i}$ 's, for $i \leq n-1$.

Theorem (Vainsencher). Let $\mathbb{P}^{N}=X_{0}$ and $X_{k}=B l_{\tilde{\Phi}_{k}} X_{k-1}$, where $\tilde{\Phi}_{k}$ denotes the strict transform of the locus of quadrics of rank at most $k$. The space of complete $(n-1)$-quadrics $X$ is isomorphic to $X \cong B l_{\tilde{\Phi}_{n-1}} X_{n-2}$.

Proof. This is a particular case of Theorem 1.2 in (15). 
It follows from this result that the points along the boundary of $X$ parametrize quadrics which are marked by another quadric over its singular locus. In other words, if $Q \in X$ is a complete quadric of rank $k$, then $Q=\left(Q^{\prime}, q\right)$ where $Q^{\prime}$ is a quadric hypersurface in $\mathbb{P}^{n}$ of rank $k$, and $q$ is a complete quadric over $\operatorname{Sing}\left(Q^{\prime}\right) \cong \mathbb{P}^{n-k}$. In this case $Q \in E_{k}$, for $1 \leq k \leq n-1$. The divisor $E_{n}$ is the strict transform of $\Phi_{n} \subset \mathbb{P}^{N}$, hence, $Q \in E_{n}$ represents a quadric of rank $n$.

\section{Example:}

Let $X$ be the space of complete 2-quadrics. A quadric of rank 1 whose marking consists of a double line with two marked points can be represented as $Q=\left(x_{0}^{2}, x_{1}^{2},\left(a x_{2}+b x_{3}\right)^{2}\right)$.

\section{Example:}

The family of $n-1$-quadrics $\mathcal{X}_{t}=\left\{x_{0}^{2}+t\left(F\left(x_{1}, \ldots, x_{n}\right)\right)\right\}$ has as its central fiber, $(t=0)$, the double $(n-1)$-plane with a marking $\mathcal{X}_{0}=\left(x_{0}^{2}, F\left(x_{1}, \ldots, x_{n}\right)\right)$.

Vainsencher's result implies that $\operatorname{Pic}(X) \otimes \mathbb{Q}$ is generated by boundary divisors $\left\langle E_{1}, \ldots E_{n}\right\rangle$. We show more; the cone of effective divisors $\operatorname{Eff}(X)$ is also generated by boundary divisors.

Proof of Theorem A. We make use of the following strategy. Let $\overline{\mathrm{NE}}(X)$ be the dual cone of $\operatorname{Nef}(X)$; this is the Mori cone of effective curves (see (6)). If the divisors $H_{i}$ are basepointfree, then $\left\langle H_{1}, \ldots, H_{n}\right\rangle \subset \operatorname{Nef}(X)$. The opposite containment is equivalent to $\left\langle H_{1}, \ldots, H_{n}\right\rangle^{\vee} \subset$ $\operatorname{Nef}(X)^{\vee} \cong \overline{\mathrm{NE}}(X)$. We show this latter statement holds by showing that the dual curves to $\left\langle H_{1}, \ldots, H_{n}\right\rangle$ are effective curves. 
The divisors $H_{i}$ are basepoint-free. In other words, given $\Lambda_{i}$ a linear subspace of dimension $i-1$ and $Q \in X$ such that $H_{i}\left(\Lambda_{i}\right)$ vanishes on $Q$, then we can find a distinct $\Lambda_{i}^{\prime}$ such that $H_{i}\left(\Lambda_{i}^{\prime}\right)$ does not vanish on $Q$. Indeed, if $Q$ is smooth or $\operatorname{dim} \operatorname{Sing}(Q)<\operatorname{codim} \Lambda_{i}$, then it is clear. If $\operatorname{dim} \operatorname{Sing}(Q) \geq \operatorname{codim} \Lambda_{i}$, then $\Lambda_{i}$ is tangent to the complete quadric $Q=\left(Q^{\prime}, q\right)$ as long as the restriction $\Lambda_{i} \mid \operatorname{Sing}(Q)$ is tangent to the marking-quadric $q$ [See, (16)]. If the marking-quadric $q$ is smooth, then $H_{i}\left(\Lambda_{i}^{\prime}\right)$ does not vanish on $Q$ if the restriction $\Lambda_{i}^{\prime} \mid \operatorname{Sing}(Q)$ is not tangent to $q$. In case the marking-quadric $q$ is singular, we repeat the previous argument for the restriction $\Lambda_{i} \mid \operatorname{Sing}(q)$. So, inductively, we can find $\Lambda_{i}^{\prime}$ such that the complete quadric $Q$ is not tangent to $\Lambda_{i}^{\prime}$, and consequently $H_{i}\left(\Lambda_{i}^{\prime}\right)$ does not vanish on $Q$. Hence, $H_{i}$ is basepoint-free and $\left\langle H_{1}, \ldots, H_{n}\right\rangle \subset \operatorname{Nef}(X)$.

Let us show the opposite containment. Consider the following flag,

$$
\mathbf{F l}_{\circ}=\left\{p t=F_{1} \subset F_{2} \subset \cdots \subset F_{n+1}=\mathbb{P}^{n}\right\}
$$

where each $F_{i}$ stands for a linear subspace of dimension $i-1$ contained in $F_{i+1}$.

Observe that a complete quadric of rank 1 whose nested markings all have rank 1 is supported on a flag $\mathbf{F} \mathbf{l}_{\circ}$. Hence, by letting the subspace $F_{i}$ vary inside $F_{i+1}$ such that it contains $F_{i-1}$, we get a rational curve $\mathbf{F l}_{i} \subset X$ for each $1 \leq i \leq n$. Observe that $\mathbf{F l}_{i} \cdot H_{j}=\delta_{i j}$. This implies that the curves $\left\langle\mathbf{F l}_{1}, \ldots, \mathbf{F} \mathbf{l}_{n}\right\rangle$ span the dual cone to $\left\langle H_{1}, \ldots, H_{n}\right\rangle$. Since the $\mathbf{F} \mathbf{l}_{i}$ are effective, the result follows. 
Let us now prove the claim about the effective cone. It is clear that $\left\langle E_{1}, \ldots E_{n}\right\rangle \subset \operatorname{Eff}(X)$. To show that this is an equality, we consider a general effective divisor $D$ and show that it can be written as a linear combination $D=a_{1} E_{1}+\cdots+a_{n} E_{n}$, where $a_{i} \geq 0$ for all $i$. In order to do that, consider the following curves which sweep out each boundary divisor $E_{k}$, for $1 \leq k \leq n-1$. Let us denote by $B_{k}$ the 1-parameter family of complete quadrics $Q=\left(Q^{\prime}, q\right) \in E_{k}$, such that $Q^{\prime}$ is fixed and the marking quadric $q \subset \mathbb{P}^{n-k} \cong \operatorname{Sing}\left(Q^{\prime}\right)$ varies in a general pencil of dual quadrics. In other words, the following intersection numbers hold,

$$
B_{k} \cdot E_{k} \leq 0 \text { and } \quad B_{k} \cdot E_{k+1}>0
$$

and zero otherwise. In fact, the number $B_{k} \cdot E_{k+1}=n-k+1$, as it is the number of times the marking quadric $q$ becomes singular. On the other hand, observe that $B_{k} \subset E_{k}$ and the normal bundle $N_{E_{k} / X} \cong \mathcal{O}(-1)$ for $1 \leq k \leq n-1$. Thus, $B_{k} . E_{k}=c_{1}\left(\mathcal{O}_{B_{k}}(-1)\right)=-(n-k)$.

Let $D=a_{1} E_{1}+\cdots+a_{n} E_{n}$ be a general effective divisor, so that it does not contain any of the curves $B_{k}$. It follows that $B_{k} . D \geq 0$, and by the inequalities $(1),(n-k) a_{k} \leq(n-k+1) a_{k+1}$ for $1 \leq k \leq n-1$. This implies that

$$
a_{1} \leq \frac{n}{n-1} a_{2} \leq \ldots \leq n a_{n}
$$

By intersecting $D$ with a general pencil $G \subset \mathbb{P}^{N}$, we get that $0 \leq a_{1}$. This finishes the proof.

The following corollary guaranties that the MMP yields finitely many models when applied to $X$. 
Corollary. The space of complete $(n-1)$-quadrics is a Mori dream space.

Proof. Vainsencher's Theorem implies that one can compute the canonical class of $X$ recursively as follows.

$$
\begin{gathered}
K_{X}=K_{X_{n-2}}+2 E_{n-1} \\
\vdots \\
K_{X_{i}}=K_{X_{i-1}}+\left(\Gamma_{i}-1\right) E_{i} \\
\vdots \\
K_{X_{1}}=K_{\mathbb{P}^{N}}+\left(\Gamma_{1}-1\right) E_{1}
\end{gathered}
$$

where $N=\left(\begin{array}{c}n+2 \\ 2\end{array}\right)-1$ and $\Gamma_{i}$ denotes the codimension of the locus $\Phi_{i} \subset \mathbb{P}^{N}$. From $(5$, p. 38), it follows that $E_{i}=2 H_{i}-H_{i-1}-H_{i+1}$ for $1<i<n$. We can write the canonical class $K_{X}$ in terms of the generators of the nef cone,

$$
K_{X}=-2 H_{1}-H_{2}-\cdots-H_{n-1}-2 H_{n}
$$

Hence, $X$ is Fano by Theorem A. In (7) it is shown (in great more generality) that any smooth Fano variety is a Mori dream space. The corollary follows.

\subsection{Cycles of codimension two on the space of complete conics}

In this section we use the techniques from the proof of Theorem A in order to study higher codimension cycles. We compute $\operatorname{Eff}^{2}(X)$, the cone of codimension-two cycles on the space of complete conics. 
Let $X$ denote the space of complete conics in $\mathbb{P}^{2}$. The space $X$ has the following stratification in $S L_{3}$-orbits. $X=U \cup\left(E^{\circ} \cup \Delta^{\circ}\right) \cup E \Delta$, where $U$ is an open dense subset, and $E^{\circ}$ and $\Delta^{\circ}$ are $S L_{3}$-orbits of codimension 1 . The unique closed orbit is $E \Delta$ [see, (5)]. The effective cone of divisors $\operatorname{Eff}(X)=\langle E, \Delta\rangle$, where $E$ parametrizes double lines with two marked-points. The divisor $\Delta$ parametrizes reducible conics. The nef cone $\operatorname{Nef}(X)=\langle H, T\rangle$, where $H$ parametrizes all the conics which pass through a fixed point, and $T$ denotes the closure of all the conics tangent to a fixed line.

\section{Cycles of Codimension Two}

Proposition. The space $A^{2}(X) \otimes \mathbb{Q}$ has dimension 3 . The effective cone is generated by the following classes

$$
\operatorname{Eff}^{2}(X)=\langle H E, T \Delta, E \Delta\rangle
$$

Proof. We make use of the following strategy. Since we know $\langle H E, T \Delta, E \Delta\rangle \subset \operatorname{Eff}^{2}(X)$, it suffices to show that the opposite containment holds. The latter containment is equivalent to the inclusion of the dual cones $\langle H E, T \Delta, E \Delta\rangle^{\vee} \subset \operatorname{Eff}^{2}(M)^{\vee}$, where by definition $\operatorname{Eff}^{2}(X)^{\vee}=$ $\operatorname{Nef}^{3}(X)$. We have thus reduced the proposition showing that the classes in $\langle H E, T \Delta, E \Delta\rangle^{\vee}$ are nef. This is what we prove next.

Let us compute the cone $\langle H E, T \Delta, E \Delta\rangle^{\vee}$. This dual cone consists of all the classes $\alpha \in$ $A_{2}(X)$ such that $\alpha \cdot \beta \geq 0$, where $\beta \in\langle H E, T \Delta, E \Delta\rangle$. This intersection, in terms of the basis $\left\langle H^{2} E, T^{2} \Delta, H^{3}\right\rangle$ for $A_{2}(X)$, reads as follows. We need $a, b, c \in \mathbb{Q}$ such that $\left(a H^{2} E+b T^{2} \Delta+\right.$ $\left.c H^{3}\right)(l H E+r T \Delta+s E \Delta) \geq 0$, where $r, l, s \geq 0$. We can split this inequality in three, 


$$
\begin{aligned}
H E . \alpha & =H E\left(a H^{2} E+b T^{2} \Delta+c H^{3}\right) \\
& =a 0+12 b+0 c \geq 0 \\
T \Delta . \alpha & =T \Delta\left(a H^{2} E+b T^{2} \Delta+c H^{3}\right) \\
& =8 a+0 b+6 c \geq 0, \text { and } \\
E \Delta . \alpha & =E \Delta\left(a H^{2} E+b T^{2} \Delta+c H^{3}\right) \\
& =-8 a-8 b+0 c \geq 0 .
\end{aligned}
$$

From these three inequalities, we have that the cone $\langle H E, T \Delta, E \Delta\rangle^{\vee}$ is generated by the following three classes,

$$
\begin{aligned}
& \alpha_{1}=3 H^{2} E-4 H^{3}, \\
& \alpha_{2}=-3 H^{2} E+3 T^{2} \Delta+4 H^{3}, \text { and } \\
& \alpha_{3}=H^{3} .
\end{aligned}
$$

Using the equations $E=2 H-T, \Delta=2 T-H$, and $2 H^{3}-3 H^{2} T+3 H T^{2}-2 T^{3}=0$, which is the linear relation imposed in $A_{2}(X)$, we have

$$
\begin{aligned}
\alpha_{1} & =3 H^{2} E-4 H^{3}, \\
& =2\left(2 H^{3}-3 H^{2} T\right), \\
& =2(H+T) E \Delta,
\end{aligned}
$$

and 


$$
\begin{aligned}
\alpha_{2} & =-3 H^{2} E+3 T^{2} \Delta+4 H^{3} \\
& =4 T^{3} .
\end{aligned}
$$

Since $H^{3}$ and $T^{3}$ are nef classes, the theorem follows if the class $\tau=(H+T) E \Delta$ is nef. In other words,

$$
\operatorname{Eff}^{2}(X)=\langle H E, T \Delta, E \Delta\rangle \quad \text { if and only if } \tau \text { is a nef class. }
$$

This is proved in the next lemma, which completes the proof of the proposition.

Lemma. The class $\tau=(H+T) E \Delta \in A_{2}(X)$ is nef.

Proof. Throughout this proof $Z$ denotes an irreducible threefold. Recall $X$ is stratified in $S L_{3} \mathbb{C}$-orbits with desirable properties (5). The proof is carried out by analyzing all the cases in which an irreducible threefold $Z$ intersects the $S L_{3}$-strata. The first case is the following. If $Z$ intersects all the strata transversally, then by Kleiman's Transversality Theorem, the intersection $Z . \tau$ is generically transversal, $\operatorname{dim} Z . \tau=0$, and the result follows. However, the intersection of $Z$ with $\tau$ may not be transversal, so Kleiman's Theorem does not apply directly.

Since $\tau$ is a surface in $E \cap \Delta$, it suffices to analyze the cases for $\operatorname{dim} Z \cap E$ and $\operatorname{dim} Z \cap E \cap \Delta$ listed below. The rows of the following table display the possibilities we are going to examine and re cases in which Kleiman's Theorem does not apply directly. 


\begin{tabular}{|c|cc|c|}
\hline Case & $\operatorname{dim} E \cap Z$ & $\operatorname{dim} E \cap \Delta \cap Z$ & Geometry \\
\hline$(1)$ & 2 & 1 & $\tau \in \operatorname{Nef}^{1}(E \Delta)$ \\
\hline$(2)$ & 3 & 3,2 & $\tau \in \operatorname{Nef}^{2}(M)$ \\
\hline$(3)$ & 2 & 2 & $\tau \in \operatorname{Nef}^{2}(E)$ \\
\hline
\end{tabular}

(1) Suppose now $\operatorname{dim} Z \cap E=2$ and $\operatorname{dim} Z \cap E \cap \Delta=1$. Since $H+T$ is the hyperplane section of the embedding $X \subset \mathbb{P}^{9} \times \mathbb{P}^{9 *}, \tau$ is a very ample divisor in $E \Delta$. Since $Z \cap E \Delta$ is a curve, $Z . \tau>0$.

(2) Suppose $\operatorname{dim} Z \cap E=3$ and $\operatorname{dim} Z \cap \Delta=3$. Then, $Z \subset E \Delta$ and irreducibility implies that $Z=\beta E \Delta$ for some $\beta>0$. Hence, $Z . \tau \geq 0$ follows. Secondly, suppose $\operatorname{dim} Z \cap E=3$ and $\operatorname{dim} Z \cap \Delta=2$. Since $Z$ is irreducible and the complement of the $S L_{3}$-orbit $E \backslash E^{\circ}=E \Delta$, $Z$ is a divisor in $E$. This means $Z$ can be written as linear combination of the generator for the effective divisor of $E$. Namely $Z=\alpha H E+\beta E \Delta$ where $\beta, \alpha \geq 0$. Observe now that $(H E . H+H E . T) E \Delta=0$, thus we have the following,

$$
\begin{aligned}
Z \cdot \tau & =(\alpha H E+\beta E \Delta) \cdot \tau \\
& =(\alpha H E+\beta E \Delta) \cdot(H+T) E \Delta \\
& =(\alpha H E+\beta E \Delta) \cdot(H E \Delta+T E \Delta) \\
& =\alpha E \Delta(H E \cdot H+H E \cdot T)+\beta(E \Delta \cdot H E \Delta+E \Delta \cdot T E \Delta) \\
& =(\beta E \Delta)(H+T) E \Delta \geq 0 .
\end{aligned}
$$

Note the last equality above brings us to the case in which $Z=\beta E \Delta$. 
(3) Suppose now $\operatorname{dim} Z \cap E=2$ and $\operatorname{dim} Z \cap E \Delta=2$. Observe first that $E=\mathbb{P}^{2[2]}$, the Hilbert scheme of 2 points in $\mathbb{P}^{2}$. In which case we have the Chow morphism

$$
\operatorname{ch}: \mathbb{P}^{2[2]} \longrightarrow \mathbb{P}^{2(2)}
$$

where $\mathbb{P}^{2(2)}$ is the symmetric product (singular) and $\mathbb{P}^{2[2]}$ is a desingularization. It is known that $\operatorname{Pic}(E)_{\mathbb{Q}}=\langle C, B\rangle$ where $C:=c h^{*}(h), h$ is the hyperplane section and $B$ is the exceptional divisor. This means $C$ is a nef divisor, hence $C^{2}$ is a nef cycle of codimension 2 in $E$. Therefore, $C^{2} \cdot Z^{\prime} \geq 0$ for any irreducible surface $Z^{\prime} \subset E$. Taking $Z^{\prime}=(Z \cap E)$, we can conclude that $Z^{\prime} . C^{2} \geq 0$ for any $Z^{\prime}$ (which is irreducible by Kleiman's Theorem.) Thus the result follows from the the following claim.

Claim: If we consider the codimension-two cycle $C^{2} \subset E$ as a codimension-three cycle in $M$, then

$$
8 C^{2}=\tau
$$

In order to prove this claim, it suffices to compute the class of $C^{2}$ as a codimension-three cycle of $M$. That is, we must find numbers $a, b, c \in \mathbb{Q}$ such that $C^{2}=a H^{2} E+b T^{2} \Delta+c H^{3}$. Observe $C^{2}$ admits the following geometric description,

$C^{2}=\left\{\right.$ subschemes of length 2 in $\mathbb{P}^{2}$ whose support intersects two lines $\} \quad$ (as a cycle in $E$ ) $=\left\{\right.$ double lines whose marked points intersect two lines $\left.L_{1}, L_{2}\right\} \quad($ as a cycle in $M)$. 
It follows that we have the following intersections numbers in $M$,

$$
C^{2} \cdot H^{2}=1, \quad C^{2} \cdot H T=1, \quad C^{2} \cdot T^{2}=2 .
$$

Indeed, $C^{2} \cdot H^{2}=1$ is the number of double lines containing the two points (imposed by $H^{2}$ ) and whose (unordered) marked points intersect the two lines $L_{1}, L_{1}$. The other two are similar.

This completes the proof of the lemma.

Corollary. The nef cone in codimension three is generated by the following classes $\operatorname{Nef}^{3}(X)=$ $\left\langle H^{3}, T^{3}, \alpha\right\rangle$, where $\alpha=(H+T) E \Delta$. 


\section{CHAPTER 3}

\section{MORI'S PROGRAM FOR COMPLETE $(N-1)$-QUADRICS}

In this Chapter we prove Theorem B and Theorem C. The former gives a moduli interpretation to many models $X(D)$, where $D$ is a movable divisor and $X$ is the space of complete 2-quadrics. The latter gives a modular interpretation to the model $X\left(H_{k}\right)$, where $H_{k}$ is any generator of the nef cone $\operatorname{Nef}(X)$ and $X$ denotes the space of complete $(n-1)$-quadrics.

\subsection{Mori's program in arbitrary dimensions.}

Since the entries of $\Lambda^{k} Q \in \mathbb{P}\left(S^{2}\left(\Lambda^{k} V\right)\right)$ are the $(k \times k)$-minors of $Q$, it follows that the $k$ th-projection $\rho_{k}: X \rightarrow \mathbb{P}\left(S^{2}\left(\Lambda^{k} V\right)\right)$ is a bijection over the locus of non-singular quadrics. Indeed, given two non-singular matrices $A$ and $B$, if each of the respective $(k \times k)$-minors of $A$ and $B$ are equal, then $A=\lambda B$ for some non-zero $\lambda$.

Definition 3.1. Let $Q \subset \mathbb{P}^{n}$ be a smooth quadric hypersurface. The second order Chow form $C F_{Q} \in \mathbb{P} H^{0}\left((\mathbb{G}(k, n), \mathcal{O}(2)) \subset \mathbb{P}\left(S^{2}\left(\Lambda^{k} V\right)\right)\right.$ parametrizes tangent $k$-planes to $Q$.

Lemma. Let $Q \subset \mathbb{P}^{n}$ be a smooth quadric hypersurface. The second order Chow form $C F_{Q}=$ $\Lambda^{k} Q$ is equal to the $k$-th wedge of $Q$.

Proof. Let $L \subset \mathbb{P}^{n}$ be a $k$-plane and $q=Q \mid L$ be the restriction of $Q$ to $L$. If $L$ is not contained in $Q$, then the $k$-plane $L$ is tangent to $Q$ if $q$ is singular, which is equivalent to $\operatorname{det} q=0$. 
Observe that the $k$-plane $L \in \mathbb{G}(k, n)$ belongs to the zero locus of the Chow form $C F_{Q}$ if and only if $L$ belongs to the zero locus of the quadric $\Lambda^{k} Q$. Indeed,

$$
\begin{aligned}
L^{t}\left(\Lambda^{k} Q\right) L & =\Lambda^{k}\left(L^{t} Q L\right) \\
& =\Lambda^{k} q \\
& =\operatorname{det}(q) .
\end{aligned}
$$

It follows that $\operatorname{det} q=0$ if and only if $L$ is in the zero locus of $\Lambda^{k} Q$. Hence, $C F_{Q}$ and $\Lambda^{k} Q$ define the same divisor on $\mathbb{G}(k, n)$

This lemma implies that the image of the projection $\rho_{k}: X \rightarrow \mathbb{P}\left(S^{2}\left(\Lambda^{k} V\right)\right)$ carries a moduli interpretation: it parametrizes tangent $(k-1)$-planes to complete quadrics. We define the second order Chow variety Chow $_{2}(k-1, X)$ as the image of $\rho_{k}(X) \subset \mathbb{P}\left(S^{2}\left(\Lambda^{k} V\right)\right)$.

Theorem C. Let $H_{k}$ be a generator of $\operatorname{Nef}(X)$, the nef cone of $X$. For each $1 \leq k \leq n$, the model

$$
X\left(H_{k}\right)=\operatorname{Proj}\left(\bigoplus_{m \geq 0} H^{0}\left(X, m H_{k}\right)\right) \cong \operatorname{Chow}_{2}(k-1, X)^{\nu}
$$

is isomorphic to the normalization of the second order Chow variety.

Proof. By definition $X \subset \mathbb{P}\left(S^{2}(V)\right) \times \mathbb{P}\left(S^{2}\left(\Lambda^{2} V\right)\right) \times \ldots \times \mathbb{P}\left(S^{2}\left(\Lambda^{n} V\right)\right)$, and the morphism $\rho_{k}: X \rightarrow \mathbb{P}\left(S^{2}\left(\Lambda^{k} V\right)\right)$ is the projection onto the $k$ th-factor. In order to establish that $X\left(H_{k}\right) \cong$ $\rho_{k}(X)^{\nu}$, it suffices show that both $\rho_{k}$ and the induced map $\phi_{H_{k}}: X \rightarrow X\left(H_{k}\right)$ contract the same extremal rays in $\overline{\mathrm{NE}}(X)$. (See, (6) for a proof of this fact.) By Theorem $B$, we know that $\phi_{H_{k}}$ contracts the classes $\mathbf{F l}_{j}$ for $j \neq k$, which generate the Mori cone of curves $\overline{\mathrm{NE}}(X)$. In 
order to show that the morphism $\rho_{k}$ contracts those same curve classes, we use a parametric representation of them. Let us describe such a parametrization.

This description follows (2) and (17) closely. We write a complete quadric as $Q=M^{t} q M$, where the matrix $M=\left(M_{i j}\right)$ has 1's along the diagonal, and $M_{k, k+1}=t_{k}$ are affine parameters above the diagonal, and zero otherwise. For example, $M$ has the following form in case $n=3$,

$$
M=\left(\begin{array}{cccc}
1 & t_{1} & 0 & 0 \\
& 1 & t_{2} & 0 \\
& & 1 & t_{3} \\
& & & \\
& & & 1
\end{array}\right)
$$

The matrix $q=\left[1, q_{1}, q_{1} q_{2}, \ldots, q_{1} \cdots q_{n}\right]$ is a diagonal matrix where $q_{j}$ are affine parameters. Observe that the matrix $M$ as described above and $q_{r}=0$, for $1 \leq r \leq n$, gives rise to the complete quadric

$$
Q=M^{t} q M=\left(\left(x_{0}+t_{1} x_{1}\right)^{2},\left(x_{1}+t_{2} x_{2}\right)^{2}, \ldots,\left(x_{n-1}+t_{n} x_{n}\right)^{2}\right)
$$

where the marking has rank 1.

We obtain a parametrization of the representatives for the curve classes $\mathbf{F}_{j} \in \overline{\mathrm{NE}}(X)$, when $q_{1}=\cdots=q_{n}=0$ and $t_{k}=0$ for all $k \neq j,(17)$. Hence, the parameter $t_{j}$ in the expression of $M$ is an affine parameter of the curve $\mathbf{F l}_{j}$. 
In order to conclude that $\rho_{k}$ contracts $\mathbf{F l}_{j}$ for $j \neq k$, it suffices to show that $t_{l}=0$ for $l \neq j$ (in particular $t_{k}=0$ ), make the form $\Lambda^{k} Q=\Lambda^{k} M^{t}\left(\Lambda^{k} q\right) \Lambda^{k} M$ constant. For example, consider $n=3$. From matrix

$$
\Lambda^{2} Q=\left(\begin{array}{cccccc}
1 & t_{2} & 0 & t_{1} t_{2} & 0 & 0 \\
t_{2} & 1 & 0 & t_{1} t_{2}^{2} & 0 & 0 \\
0 & 0 & 0 & 0 & 0 & 0 \\
t_{1} t_{2} & t_{1} t_{2}^{2} & 0 & t_{1}^{2} t_{2}^{2} & 0 & 0 \\
0 & 0 & 0 & 0 & 0 & 0 \\
0 & 0 & 0 & 0 & 0 & 0
\end{array}\right)
$$

it follows that $\rho_{2}$ contracts $\mathbf{F}_{1}=\left\{t_{2}=t_{3}=0\right\}$ and $\mathbf{F}_{3}=\left\{t_{1}=t_{2}=0\right\}$. The fact that $q=[1,0, \ldots, 0]$ simplifies the computation of $\Lambda^{k} Q$ in general. We omit the details since no difficulty arises. This completes the proof.

Remark. The previous result considers the projection of $X$ onto the kth-factor. One can consider other projection morphisms. For instance, let $\underline{i}=\left(i_{1}, \ldots, i_{n}\right)$, for $1 \leq i_{l}<i_{j} \leq n$. One can project $\rho_{\underline{i}}: X \rightarrow \mathbb{P}_{i_{1}} \times \cdots \times \mathbb{P}_{i_{j}}$, where $\mathbb{P}_{i_{j}}=\mathbb{P}\left(S^{2}\left(\Lambda^{i_{j}} V\right)\right)$. Assuming that $\Lambda^{0} V=\mathbb{C}$, and using this notation the projection morphisms in the previous result are of the form $\rho_{\underline{i}}$, where

$$
\underline{i}=(0, \ldots, k, \ldots, 0) .
$$


By the observation at the beginning of section 3.1, the projection maps $\rho_{\underline{i}}$ are birational morphisms. The $\rho_{\underline{i}}$ provide, in general, a number of birational models of X. A good deal of this thesis is devoted to fully understanding all these projection maps in the case when $n=3$.

\subsection{Mori's program for complete quadric surfaces}

Semple (1),(2) studied in detail the space of complete quadric surfaces $X$. The rest of this dissertation is devoted to further studying $X$ by applying the Minimal Model Program (MMP) on it.

Definition 3.2. Let $Y$ be a smooth projective variety over $\mathbb{C}$. The movable cone $\overline{\operatorname{Mov}}(Y) \subset$ $N^{1}(Y)$ is the closure of the cone generated by classes of effective Cartier divisors $L$ such that the base locus of $|L|$ has codimension at least two. We say that a divisor is movable if its numerical class lies in $\overline{\operatorname{Mov}}(Y)$.

We will be able to interpret the spaces studied by Semple as models $X(D)$ where $D \in$ $\operatorname{Nef}(X)$. This section contains the preliminaries needed to show more; we aim to describe all the models $X(D)$, where $D$ is in the movable cone $\operatorname{Mov}(X)$, and exhibit such models as moduli spaces.

Let us recall some well-known properties of the space $X$. Following (15), the space $X \cong$ $B l_{\tilde{\Phi}_{2}} X_{1}$, where $X_{1}$ is a blowup of $\mathbb{P}^{9}$ along $\Phi_{1}$, the locus of symmetric matrices of rank 1 , and $\tilde{\Phi}_{2}$ denotes the strict transform of the locus of symmetric matrices of rank 2 . We can also obtain $X$ by blowing up $\mathbb{P}^{9 *}$, the space of dual quadrics in $\mathbb{P}^{3}$, in the same manner. 
The divisor class $H_{i}$ in $\operatorname{Pic}(X)$, as defined in 1.2 , coincides with the class of the strict transform of a generator of the ideal of $\Phi_{i} \subset \mathbb{P}^{9}$. Indeed, let us denote by $p: X \rightarrow \mathbb{P}^{9}$ the blowup map, clearly $p^{*}\left(\mathcal{O}_{\mathbb{P}^{9}}(1)\right)=H_{1}$. Moreover, let $h_{1}$ and $h_{2}$ be two generators of the ideals $I\left(\Phi_{1}\right)$ and $I\left(\Phi_{2}\right)$, respectively. Since

$$
\begin{aligned}
& p^{*}\left(\left[h_{1}\right]\right)=2 H_{1}-E_{1}, \\
& p^{*}\left(\left[h_{2}\right]\right)=3 H_{1}-2 E_{1}-E_{2},
\end{aligned}
$$

in $\operatorname{Pic}(X)$, we can compare these classes with those of $H_{2}$ and $H_{3}$.

Lemma. Let $\mathrm{H}_{2}, \mathrm{H}_{3}$ be the divisors as defined in 1.2. Their classes in $\mathrm{Pic}(X)$ are

$$
\begin{aligned}
& H_{2}=2 H_{1}-E_{1}, \\
& H_{3}=3 H_{1}-2 E_{1}-E_{2} .
\end{aligned}
$$

Proof. Let $G, C_{2}, L_{2} \subset X$ be the following test curves. The curve $G$ stands for a general pencil, $C_{2}$ is defined by the product of a fixed plane $P_{0}$ and a pencil of planes $P_{t}$ such that $C_{2}=\left\{P_{0} P_{t}\right\}$. The curve $L_{2}$ is defined by fixing two planes whose intersection is the line $l$ and letting one of the two marked points on $l$ vary. 
The following numbers determine the class of $H_{i}$ for $i=2,3$.

$$
\begin{array}{lll}
G \cdot H_{1}=1 & C_{2} \cdot H_{1}=1 & L_{2} \cdot H_{1}=0 \\
G \cdot H_{2}=2 & C_{2} \cdot H_{2}=0 & L_{2} \cdot H_{2}=0 \\
G \cdot H_{3}=3 & C_{2} \cdot H_{3}=0 & L_{2} \cdot H_{3}=1 \\
G \cdot E_{1}=0 & C_{2} \cdot E_{1}=2 & L_{2} \cdot E_{1}=0 \\
G \cdot E_{2}=0 & C_{2} \cdot E_{2}=-1 & L_{2} \cdot E_{2}=-1
\end{array}
$$

The normal bundle $N_{E_{2} \backslash X} \cong \mathcal{O}_{E_{2}}(-1)$. The restriction to the generic line $L_{2} \subset E_{2}$ is isomorphic to $\mathcal{O}_{\mathbb{P}^{1}}(-1)$, hence $L_{2} \cdot E_{2}=-1$. Similarly $C_{2} \cdot E_{2}=-1$. If we write $H_{i}=a H_{1}+b E_{2}+c E_{3}$ for $i=2,3$, and use the test curves $G, C_{2}, L_{2}$ to find the values of $a, b, c$, then the result follows.

In the following proposition, we denote by $H_{1}$ the pull-back of $\mathcal{O}_{\mathbb{P}^{9}}(1)$ and $H_{2}$ the pull-back of a generator of the ideal $I\left(\Phi_{1}\right)$. The information provided by this proposition is put into context in section 3.5 and Chapter 4.

Proposition. If $X_{1}=B l_{\Phi_{1}} \mathbb{P}^{9}$, then the nef cone is generated by $\operatorname{Nef}\left(X_{1}\right) \cong\left\langle H_{1}, H_{2}\right\rangle$.

Proof. $H_{1}$ is clearly an extremal ray of the nef cone. The divisor $H_{2}$ is basepoint-free by definition and we have that $H_{2} . S=0$, where $S$ denotes a pencil $Q_{1}+t Q_{1}^{\prime}$ of quadrics of rank 1 . Since the curve $S$ sweeps out the secant variety $\operatorname{Sec}\left(\Phi_{1}\right)=\Phi_{2}, H_{2}$ induces a small contraction $\phi_{1}: X_{1} \rightarrow Z$ 
The canonical divisor $K_{X_{1}}=-10 H_{1}-5 E_{1}=-5 H_{2}$. Hence, $K_{X_{1}} \cdot S=0$ and $X_{1}$ is a flop of a space $Y_{1}$ over $Z$ in the following diagram.

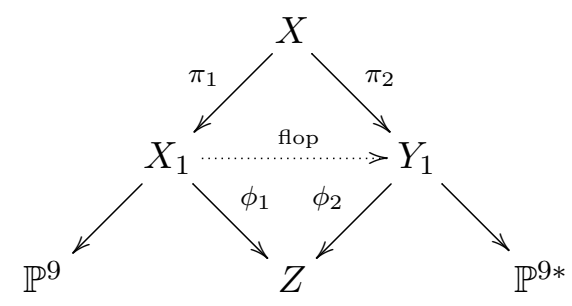

where $X_{1}$ is as above, and $Y_{1}=B l_{\Phi_{1}} \mathbb{P}^{9 *}$. The morphism $\phi_{1}$ is induced by the sub-linear series of $(2 \times 2)$-minors cutting out $\Phi_{1} \subset \mathbb{P}^{9}$ scheme-theoretically. Observe that the both morphisms $\pi_{1}$ and $\pi_{2}$ contract the divisor $E_{2}$.

The following divisor class, and the model induced by it, was not analyzed in (2). To the best of our knowledge, this constitutes new information about the birational geometry of $X$.

Definition 3.3. Let $P$ denote the closure in $X$ of smooth quadrics $Q$ such that the induced 2-plane $\Lambda_{Q} \subset \mathbb{P}^{5}$ by one of the rulings of $Q$ has non-empty intersection with a fixed 2-plane in the Plucker embedding of $\mathbb{G}(1,3)$.

Lemma. The divisor class of $P$ is

$$
[P]=2\left(2 H_{1}-H_{2}+2 H_{3}\right)
$$

Proof. The assertion follows from the following intersection numbers

$$
P . C_{1}^{*}=0, \quad P . R_{2}=4, \quad P . C_{3}=0,
$$


where the curves $C_{1}^{*}, R_{2}, C_{3}$ are defined as follows. The curve $C_{1}^{*}$ is defined as a double plane with a pencil of dual conics on it. Let $R_{2}$ denote the strict transform to $X$ of the pencil $Q_{2}+\lambda Q_{2}^{\prime}$, where $Q_{2}$ and $Q_{2}^{\prime}$ denote quadrics of rank 2 in $\mathbb{P}^{3}$. The curve class $C_{3}$ is defined by a cone over a general pencil of conics.

Let us compute the intersection number P.R . Since $R_{2} \cdot E_{2}=2$ and $R_{2} \cdot E_{1}=R_{2} \cdot E_{3}=0$, it induces a 2-fold cover of curves $\gamma(\lambda) \rightarrow R_{2}(\lambda)$ where $\gamma(\lambda)$ represents the curve of 2-planes induced by the pencil $R_{2}$. Indeed, for each $\lambda$, the lines contained in the complete quadric quadric $Q(\lambda) \in R_{2}(\lambda)$ form two curves $C_{\lambda}, C_{\lambda}^{\prime}$ in the Grassmannian $\mathbb{G}(1,3)$. This is the Fano variety of lines $F_{1}(Q)$ (or a flat limit of it). Each of the curves $C_{\lambda}$ is contained in a unique 2-plane $\Lambda_{C_{\lambda}} \subset \mathbb{P}^{5}$. Consequently, P. $R_{2}=\operatorname{deg}(\gamma)$ as a subvariety of $\mathbb{G}(2,5)$. On the other hand, the class of the surface $S$ that a curve $C_{\lambda}$ sweeps out in the Grassmannian $\mathbb{G}(1,3)$ (as we vary $\lambda)$, is $[S]=\sigma_{2}+\sigma_{1,1} \in A_{2}(\mathbb{G}(1,3))$. Thus,

$$
\begin{array}{rlr}
P . R_{2} & =\operatorname{deg} \gamma & \text { in } \mathbb{G}(2,5) \\
& =2 S . \sigma_{1}^{2} & \\
& =2\left(\sigma_{1,1}+\sigma_{2}\right) \sigma_{1}^{2} \quad \text { in } \mathbb{G}(1,3) \\
& =4
\end{array}
$$

The numbers $P . C_{1}^{*}=0$ and $C_{3} . P=0$ follow from the fact that all the conics induced by them lie in a fixed plane. 


\subsection{Stable base locus decomposition}

In this section, we compute the stable base locus decomposition of $\operatorname{Eff}(X)$. In order to do that, we need curve classes and their intersection numbers with divisors. We summarize the intersection numbers in the following table, then describe each curve class.

\begin{tabular}{|c|c|c|c|c|c|c|c|} 
Curve class & $C . H_{1}$ & $C . H_{2}$ & $C . H_{3}$ & $C . E_{1}$ & $C . E_{2}$ & $C . E_{3}$ & Deformations \\
\hline$G$ & 1 & 2 & 3 & 0 & 0 & 4 & $X$ \\
$G^{*}$ & 3 & 2 & 1 & 4 & 0 & 0 & $X$ \\
$C_{1}$ & 0 & 1 & 2 & -1 & 0 & 3 & $E_{1}$ \\
$C_{1}^{*}$ & 0 & 2 & 1 & -2 & 3 & 0 & $E_{1}$ \\
$C_{2}$ & 1 & 0 & 0 & 2 & -1 & 0 & $E_{2}$ \\
$C_{3}$ & 1 & 2 & 0 & 0 & 3 & -2 & $E_{3}$ \\
$C_{1,2}$ & 0 & 1 & 0 & -1 & 2 & -1 & $E_{1} \cap E_{3}$ \\
$L_{2}$ & 0 & 0 & 1 & 0 & -1 & 2 & $E_{2}$
\end{tabular}

Let $G$ (respectively, $G^{*}$ ) denote the curve defined by the strict transform to $X$ of a general pencil in $\mathbb{P}^{9}$ (respectively, $\left.\mathbb{P}^{9 *}\right)$. The curve $C_{1}$ (respectively, $C_{1}^{*}$ ) is defined by considering a general pencil of conics (respectively, dual conics) on a fixed double plane. The curve $C_{2}$ is defined as the product of a fixed plane $P_{0}$ and a pencil of planes $P_{t}$ such that the marking is fixed. Let $C_{3}$ be the curve defined by the cone over the pencil of conics in a fixed plane. Let 
$C_{1,2}$ denote the curve defined by a pencil of rank 2 conics on a double plane. Such a pencil of conics is a fixed line $l_{0}$ and a pencil of lines whose base-locus is on $l_{0}$. Similarly, the curve $L_{2}$ is defined by fixing two planes whose intersection is the line $l$ and letting one of the two marked points on $l$ vary.

Notation. We denote by $C\left(H_{1}, \bar{P}\right)$ the positive linear combinations $a H_{1}+b P$ such that $0 \leq a$ and $0<b$

Proposition. The stable base locus decomposition partitions the effective cone of $X$ into the following chambers:

(1) In the closed cone spanned by non-negative linear combinations of $H_{1}, H_{2}$ and $H_{3}$, the stable base locus is empty.

(2) In the domain bounded by $H_{1}, H_{3}$ and $P$ union $c\left(H_{1}, \bar{P}\right) \cup c\left(H_{3}, \bar{P}\right)$, the stable base locus is $E_{1} \cap E_{3}$ and consists of double planes marked with a rank 2 singular conic.

(3) In the domain bounded by $H_{3}, E_{3}$ and $P$ union $c\left(H_{3}, \bar{E}_{3}\right) \cup c\left(P, \bar{E}_{3}\right)$, the stable base locus consists of the divisor $E_{3}$.

(4) In the domain bounded by $H_{1}, E_{1}$ and $P$ union $c\left(H_{1}, \bar{E}_{1}\right) \cup c\left(P, \bar{E}_{1}\right)$, the stable base locus consists of the divisor $E_{1}$.

(5) In the domain bounded by $P, E_{1}$ and $E_{3}$ union $c\left(E_{1}, E_{3}\right)$, the stable base locus consists of the union $E_{1} \cup E_{3}$.

(6) In the domain bounded by $H_{3}, E_{3}$ and $E_{2}$ union $c\left(E_{3}, E_{2}\right)$, the stable base locus consists of the union $E_{3} \cup E_{2}$. 
(7) In the domain bounded by $H_{1}, E_{1}$ and $E_{2}$ union $c\left(E_{1}, E_{2}\right)$, the stable base locus consists of the union $E_{1} \cup E_{2}$.

(8) In the domain bounded by $H_{1}, H_{2}, H_{3}$ and $E_{2}$ union $c\left(H_{1}, \bar{E}_{2}\right) \cup c\left(H_{3}, \bar{E}_{2}\right)$, the stable base locus consists of the divisor $E_{2}$.

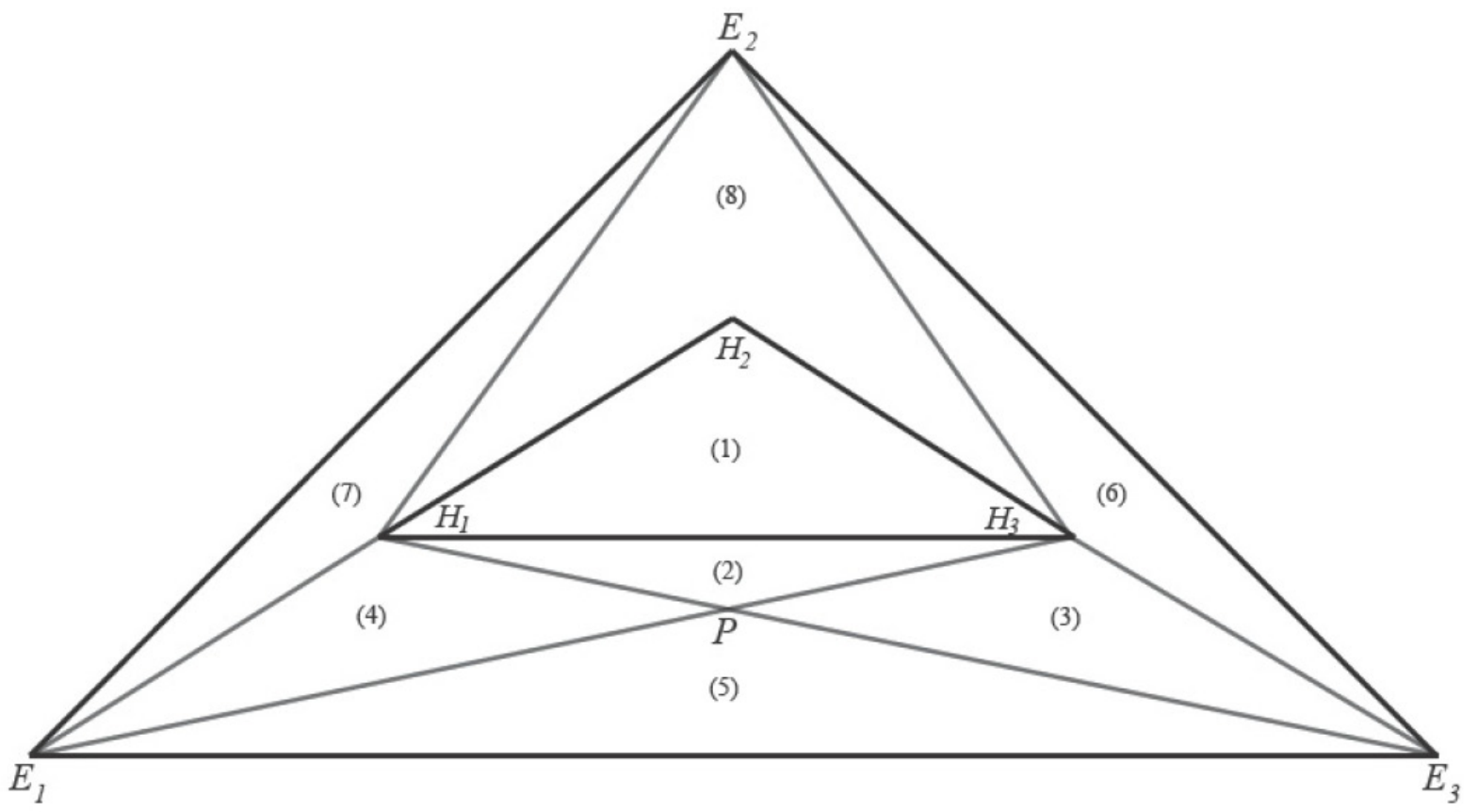

Figure 1. Stable base locus decomposition of $\operatorname{Eff}(X)$. 
Proof. We will make use of the symmetry induced by the map $\xi: X \rightarrow X$ defined by sending the quadric $Q$ to $\Lambda^{3} Q$,

$$
\xi: Q \longmapsto \Lambda^{3} Q
$$

Note that $\xi$ maps $E_{1}$ (respectively, $H_{1}$ ) to $E_{3}$ (respectively, $H_{3}$ ) and keeps $E_{2}$ (respectively, $H_{2}$ ) fixed. The stable base locus of the divisor $\xi^{*}(D)$ is equal to the inverse image under $\xi$ of the stable base locus of $D$. The symmetry given by $\xi$ will simplify our calculations.

By Theorem B, any divisor contained in the closed cone generated by $H_{1}, H_{2}$, and $H_{3}$ is basepoint-free, hence its (stable) base locus is empty.

Let $D$ be a general divisor $D=a H_{1}+b H_{2}+c H_{3}$. Consider the curves $C_{1}$ and $C_{3}$ as defined above. Then,

$$
C_{1} \cdot D=b+2 c, \quad C_{3} \cdot D=a+2 b
$$

Since the curve $C_{1}$ (respectively, $C_{3}$ ) covers $E_{1}$ (respectively, $E_{3}$ ), it follows that $E_{1}$ (respectively, $\left.E_{3}\right)$ is in the base locus of any divisor $D$ satisfying $b+2 c<0$ (respectively, $a+2 b<0$ ).

On the other hand, $\xi$ maps the plane $b+2 c=0$ to the plane $b+2 a=0$. Consequently, $E_{3}$ is in the base locus of any divisor satisfying $b+2 a<0$. Similarly, $E_{1}$ is in the base locus of the linear system $|D|$ if $c+2 b<0$. We conclude that $E_{1}$ is in the base locus of any divisor contained in the region bounded by $E_{1}, E_{2}, H_{1}$ and $E_{3}$. Similarly, $E_{3}$ is in the base locus of any divisor contained in the region bounded by $E_{3}, E_{2}, H_{3}$ and $E_{1}$. 
Now let the curve classes $C_{2}$ and $L_{2}$ be as defined above. We have the following intersection numbers:

$$
C_{2} \cdot D=a \text {, and } \quad L_{2} \cdot D=c
$$

Since both the curves $C_{2}$ and $L_{2}$ cover the divisor $E_{2}$, then $E_{2}$ is in the base locus of any divisor $D$ satisfying $a<0$ as well as $c<0$. The inequality $a<0$, tells us that $E_{2} \cup E_{3}$ is in the base locus of any divisor in the region bounded by $E_{3}, H_{3}$ and $E_{2}$ unioned with $c\left(E_{3}, \bar{E}_{2}\right) \cup c\left(H_{3}, \bar{E}_{2}\right)$. Similarly, the union $E_{2} \cup E_{1}$ is in the base locus of any divisor in the region bounded by $E_{1}, E_{2}, H_{1}$ union $c\left(E_{1}, \bar{E}_{2}\right) \cup c\left(H_{1}, \bar{E}_{2}\right)$. By intersecting these two regions, the union $E_{3} \cup E_{1}$ is in the base locus in the region bounded by $E_{1}, P$ and $E_{3}$ union $c\left(E_{1}, E_{3}\right)$.

By equation (5) above, $E_{3}$ is in the base locus in the region bounded by $E_{3}, H_{3}$ and $P$, union $c\left(P, \bar{E}_{3}\right) \cup c\left(H_{3}, \bar{E}_{3}\right)$. Symmetry implies that $E_{1}$ is in the base locus in the region bounded by $P, H_{1}$ and $E_{1}$ union $c\left(P, \bar{E}_{1}\right) \cup c\left(H_{1}, \bar{E}_{1}\right)$.

Let $C_{1,2}$ be the curve as defined above. We have that

$$
C_{1,2} \cdot D=b
$$

Since deformations of $C_{1,2}$ cover the intersection $E_{1} \cap E_{3}$, this locus $E_{1} \cap E_{3}$ is in the base locus of any divisor contained in the region bounded by $H_{1}, H_{3} P$ and $c\left(H_{1}, \bar{P}\right) \cup c\left(H_{3}, \bar{P}\right)$. Finally, $E_{2}$ is in the base locus for any divisor $|D|$ in the region bounded by $H_{1}, H_{2}, H_{3}$ and $E_{2}$. This completes the description of the base locus decomposition of $\operatorname{Eff}(X)$. 
In order to finish the proof, we need to show that the stable base locus does not get any bigger than our description of it above.

(i) The divisors $H_{1}, H_{2}, H_{3}$ are basepoint-free by Theorem B. Hence, for divisors contained in the closed cone generated by $H_{1}, H_{2}, H_{3}$ the base locus is empty.

(ii) Since $H_{1}$ and $H_{3}$ are basepoint-free, it follows that for any divisor $D$ in the interior of the cone generated by $H_{1}, H_{2}$, and $P$ the base locus of the linear system $|D|$ is contained in that of $|P|$. The same applies for the walls $c\left(H_{1}, \bar{P}\right)$ and $c\left(H_{3}, \bar{P}\right)$. Observe that the base locus of $|P|$ is the locus in $X$ parametrizing those complete quadric surfaces whose rulings induce a double line in $\mathbb{G}(1,3)$. Indeed, for any complete quadric $Q$ inducing either rank 3 or 2 conics in $\mathbb{G}(1,3)$, there is a unique 2-plane in $\mathbb{P}^{5}$ containing them. The indeterminacy of $|P|$ does not get bigger because for any pair of 2 -planes $\Lambda_{i}(\mathrm{i}=1,2)$ in $\mathbb{P}^{5}$ we can find another 2-plane missing them both. It follows that for $Q$ a quadric defining a 2-plane $\Lambda \subset \mathbb{P}^{5}$, there is a $D \in|P|$, such that $D$ does not vanish at $Q$. We conclude that the quadrics inducing double lines in $G(2,4)$ are in the base locus of $P$ i.e., $E_{1} \cap E_{3}$.

(iii-iv) Since $P$ can be written as $P=E_{3}+2 H_{1}=E_{1}+2 H_{3}$, it follows that for any divisor $D$ contained in the interior of the cone of $E_{3}, H_{3}$ and $P$ or along the wall $c\left(H_{3}, \bar{P}\right)$, the base locus of $D$ must be contained in $E_{3}$. Similarly for any divisor $D$ contained in the interior of the cone of $E_{1}, H_{1}$ and $P$ or along the walls $c\left(H_{1}, \bar{P}\right)$, the base locus of $D$ must be contained in $E_{1}$.

(v) From the previous argument, any divisor $D$ in the interior of the cone $E_{1}, E_{3}$ and $P$ the base locus must be contained in $E_{1} \cup E_{3}$.

(vi-vii) Follows easily from what we said above. 
(viii) Any divisor $D$ in the interior of the cone generated by $H_{1}, H_{3}$ and $E_{2}$ the base locus of $D$ must be contained in $E_{2}$. However, since we know the nef cone, then the base locus of any divisor in the complement of nef cone must be contained in $E_{2}$. This completes the proof.

Corollary. The movable cone $\operatorname{Mov}(X)$ of $X$ is the closed cone spanned by non-negative linear combinations of $\mathrm{H}_{1}, \mathrm{H}_{2}, \mathrm{H}_{3}$ and $P$.

\subsection{Birational models of complete quadric surfaces}

In this section we describe some birational models of the space $X$. We present the results very explicitly at the risk of making proofs longer than optimal. This approach will exhibit the moduli structure on the birational models constructed.

\section{Second Order Chow Variety $\operatorname{Chow}_{2}(1, X)$}

We define the second order Chow variety $\mathbf{C h o w}_{2}(1, X)$ as the parameter space of tangent lines to complete quadric surfaces. More precisely, let $Q \in X$ be a smooth complete quadric and let $T Q$ denote the set of tangent lines to it in the Grassmannian $\mathbb{G}=\mathbb{G}(1,3)$. Since the class $[T Q]=2 \sigma_{1} \in A^{1}(\mathbb{G})$, it follows that the subvariety $T Q$ is defined by an element in the linear system $\left|\mathcal{O}_{\mathbb{G}}(2)\right|$. Consequently, we have a map $Q \mapsto T Q \in \mathbb{P} H^{0}(\mathbb{G}, \mathcal{O}(2)) \cong \mathbb{P}^{19}$. The subvariety $T Q$ is called a quadric line-complex.

Lemma. Let $X^{\circ} \subset X$ be the open subset parameterizing smooth quadric surfaces. Then, we have an embedding

$$
\phi: X^{\circ} \rightarrow \mathbb{P}\left(H^{0}(\mathbb{G}, \mathcal{O}(2)) \cong \mathbb{P}^{19}\right.
$$


by mapping a smooth quadric $Q \mapsto T Q$ to its associated degree 2 hypersurface $T Q \subset \mathbb{G}(1,3)$.

Proof. Let $Q$ and $Q^{\prime}$ be two distinct smooth quadrics. Then there exists a point $x \in Q$ which is not in $Q^{\prime}$. The tangent space $T_{x} Q$ contains a 1-parameter family of lines tangent to $Q$ among which only 2 are also tangent to $Q^{\prime}$. This says that $T Q \neq T Q^{\prime}$ as desired. The fact that $\phi$ is a morphism is clear as we can write down the equations defining it, and that is precisely what we do next.

Proposition. The map $\phi$ extends to a morphism $\rho_{2}: X \rightarrow \mathbf{C h o w}_{2}(1, X)$.

Proof. By Serre's criteria (18, p. 283) the rational map $\phi$ extends to a complement of a subset of codimension 2. Furthermore, the space $X$ is stratified by $S L_{4}$-orbits as follows. There is an open dense orbit $X^{\circ}$, codimension 1 and 2 orbits $E_{i}^{\circ}$ and $E_{i}^{\circ} \cap E_{j}^{\circ}(i \neq j)$ respectively, and a unique closed orbit $E_{1} \cap E_{2} \cap E_{3}$. Therefore, the result follows if the map $\phi$ extends to each of the $E_{i}$ 's, i.e., $\phi\left(E_{i}\right)$ is well-defined for $i=1,2,3$.

Let us show that the map $\phi:\left(X^{\circ}\right) \rightarrow \mathbb{P}^{19}$ extends to the divisor $E_{1}$ by performing the explicit computation. First, we exhibit the extension of the map $\phi$ to the open $S L_{4} \mathbb{C}$-orbit $E_{1}^{\circ}$.

To simplify the computations, let us assume $Q_{t} \subset \mathbb{P}^{3}$ is the family $Q_{t}=\left\{x^{2}+t\left(a y^{2}+b y z+\right.\right.$ $\left.\left.c y w+d z^{2}+e z w+f^{2} w^{2}\right)=0\right\}$. The limit as $t \rightarrow 0$ is a complete quadric $\left(Q_{0}, q[y: z: w]\right) \in E_{1}$.

We compute the Chow form $T Q_{t}$ from the definition. A line in $l \subset \mathbb{P}^{3}$ is the image of the morphism

$$
[\alpha, \beta] \stackrel{g}{\longmapsto}\left[a_{1} \alpha+b_{1} \beta: \cdots: a_{4} \alpha+b_{4} \beta\right] \in \mathbb{P}^{3}
$$


The line $l$ is tangent to a quadric $Q$ as long as the the restriction of $Q$ to $l$ consists of a single point (with multiplicity two). Therefore, the discriminant $B^{2}-4 A C=0$ of the quadratic polynomial in $[\alpha, \beta]$,

$$
\begin{aligned}
g^{*} Q_{t} & =A \alpha^{2}+B \alpha \beta+C \beta^{2} \\
& =\left(a_{1}^{2}+t\left(a a_{2}^{2}+b a_{2} a_{3}+\cdots+f a_{4}^{2}\right)\right) \alpha^{2}+ \\
& +\left(2 a a_{2} b_{2}+t\left(\left(a_{2} b_{3}+a_{3} b_{2}\right) b+\cdots\right) \alpha \beta+\left(b_{1}^{2}+t\left(a b_{2}^{2}+\cdots\right)\right) \beta^{2}\right.
\end{aligned}
$$

describes the equations desired, which in Plücker coordinates is

$$
T Q_{t}=\left\{a p_{0}^{2}+b p_{0} p_{1}+d p_{1}^{2}+c p_{0} p_{2}+e p_{1} p_{2}+f p_{2}^{2}+t(\text { extra terms })=0\right\}
$$

This shows that $\phi: X^{\circ} \rightarrow \mathbb{P}^{19}$ can be extended to the whole of $E_{1}$. Similar computations show that there are extensions to all of $E_{2}$ and $E_{3}$. Indeed, Since $E_{2}$ is $S L_{4} \mathbb{C}$-invariant, then we can assume that $Q_{t}=x y+t\left(a z^{2}+b z w+c w^{2}\right)$ and analyze the normal directions at this point. Following the same argument as above, we find that the associated hypersurface, in Plücker coordinates is

$$
\Sigma_{1}\left(Q_{t}\right)=\left\{p_{0}^{2}+t(\text { other terms })=0\right\}
$$

It follows that the (radical of the) limit as $t \rightarrow 0$ coincides with the Schubert cycle $\Sigma_{1}(L) \subset$ $\mathbb{G}(1,3)$ where $L=\{x=y=0\}$. This gives the natural extension for $\phi_{\mid E_{2}}$ as desired. The case for $E_{3}$ is clear. This completes the proof. 
Semple's notation for $X\left(H_{2}\right)$ is $C_{9}^{92}[19]$. He showed $\left(2\right.$, p. 283) that $\rho_{2}(X)$ is normal. Hence, the following result follows for Theorem B.

Corollary. The morphism $\rho_{2}: X \rightarrow \mathbf{C h o w}_{2}(1, X) \subset \mathbb{P}^{19}$ contracts the divisor $E_{2}$. Furthermore $X\left(H_{2}\right) \cong \operatorname{Chow}_{2}(1, X)$.

Remark. The degree of $\mathbf{C h o w}_{2}(1, X) \subset \mathbb{P}^{19}$ has enumerative significance. It is the number of smooth quadric hypersurfaces in $\mathbb{P}^{3}$ which are tangent to 9 fixed lines in general position. The number of such lines is 92 .

\section{The Flip of $X$}

We now aim at constructing the flip $X^{+}$of the space of complete quadric surfaces. We will do so by analyzing a $\mathbb{Z} / 2$-action on $\mathbf{H i l b}^{2 x+1}(\mathbb{G}(1,3))$.

Definition 3.4. Let $f: X \rightarrow Y$ be a proper birational morphism such that the exceptional set $\operatorname{Exc}(f)$ has codimension at least two in $X$. Assume furthermore that $K_{X}$ is $\mathbb{Q}$-Cartier and $-K_{X}$ is $f$-ample. A variety $X^{+}$together with a proper birational morphism $f^{+}: X^{+} \rightarrow Y$ is called a flip of $f$ if

(1) $K_{X^{+}}$is $\mathbb{Q}$-Cartier,

(2) $K_{X^{+}}$is $f^{+}$-ample, and

(3) the exceptional set $\operatorname{Exc}\left(f^{+}\right)$has codimension at least two in $X^{+}$.

Definition 3.5. Let $\mathbf{H i l b}=\mathbf{H i l b}^{2 x+1}(\mathbb{G}(1,3))$ denote the Hilbert scheme parametrizing sub schemes of $\mathbb{G}(1,3) \subset \mathbb{P}^{5}$ whose Hilbert polynomial is $P(x)=2 x+1$. 
Proposition. Let Hilb be as defined above, then

$$
\text { Hilb } \cong B l_{\mathbb{O G}}(\mathbb{G}(2,5))
$$

where $\mathbb{O} \mathbb{G} \subset \mathbb{G}(2,5)$ denotes the Orthogonal Grassmannian inside the Grassmannian of 2-planes in $\mathbb{P}^{5}$.

Proof. Observe that a generic smooth curve with Hilbert polynomial $P(x)=2 x+1$ in $\mathbb{P}^{5}$ is a plane conic $C$. Thus, its ideal $I_{C} \subset k\left[p_{0}, \ldots, p_{5}\right]$ is generated by a quadric $F$ and three independent linear forms $L_{1}, L_{2}, L_{3}$. Since $C \subset \mathbb{G}=\mathbb{G}(1,3)$, the equation $F$ is the quadric corresponding to the Grassmannian $\mathbb{G} \subset \mathbb{P}^{5}$ under the Plücker embedding. This description gives rise to a rational map

$$
f: \mathbb{G}(2,5) \rightarrow \text { Hilb }
$$

by assigning the 2-plane $P$ defined by the independent linear forms $\left(L_{1}, L_{2}, L_{3}\right)$ to the ideal $\left\langle L_{1}, L_{2}, L_{3}\right\rangle+\langle F\rangle \subset k\left[p_{0}, \ldots, p_{5}\right]$. Observe that the exceptional locus of $f$ consists of planes in $\mathbb{P}^{5}$ such that there is a containment of ideals $\langle F\rangle \subset\left\langle L_{1}, L_{2}, L_{3}\right\rangle$ i.e., planes $P$ which are contained in the quadric $\mathbb{G} \subset \mathbb{P}^{5}$. We denote the locus parametrizing such planes by $\mathbb{O} \mathbb{G}$. This locus is precisely the Orthogonal Grassmannian. Now, we resolve the rational map $f$,

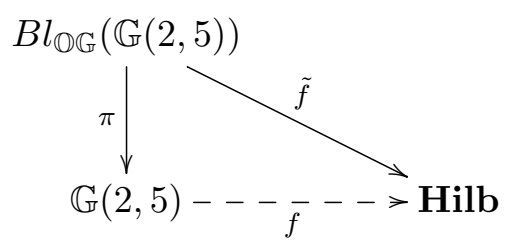


The morphism $\tilde{f}$ is an isomorphism. Indeed, the rational map $f$ is birational as it has an inverse morphism $g: \mathbf{H i l b} \rightarrow \mathbb{G}(2,5)$ defined as follows: let $[C] \in \mathbf{H i l b}$ be a generic element, then the ideal $I(C)=(F)+$ (plane) $\stackrel{g}{\mapsto}$ (plane) $\in \mathbb{G}(2,5)$. It is clear that $f \circ g=I d$, hence $f$ and consequently $\tilde{f}$ are birational. Furthermore, $\tilde{f}$ is a bijection. Indeed, since the exceptional divisor $E \subset B l_{\mathbb{G O}}(\mathbb{G}(2,5))$ is a $\mathbb{P}^{5}$-bundle over $\mathbb{O} \mathbb{G}$, then we can write $p=(P, C)$ where $P \subset \mathbb{P}^{5}$ is a 2-plane and $C \subset P$ is a plane conic. Thus, Zariski's Main Theorem implies that $\tilde{f}$ is an isomorphism.

Corollary. Let Hilb be as above, then $\operatorname{Pic}($ Hilb $) \cong\left\langle H^{+}, E_{2}^{+}, E_{1,2}^{+}\right\rangle$where $H^{+}$is the pullback of $\sigma_{1} \in A(\mathbb{G}(2,5))$ and the $E^{+}$'s are the exceptional divisors of the blowup.

Proof. The orthogonal Grassmannian $\mathbb{O} G$ has two components, hence the result follows.

If the field $k$ is algebraically closed, then for a given a smooth quadric $Q \subset \mathbb{P}^{3}$, the Fano variety of lines $F_{1}(Q) \subset \mathbb{G}(1,3)$ consists of two smooth conics. By exchanging such conics we get a $\mathbb{Z} / 2$-action on $\mathbf{H i l b}^{2 x+1}(\mathbb{G}(1,3))$.

Lemma. There is a nontrivial globally defined $\mathbb{Z} / 2$-action on $\mathbf{H i l b} \mathbf{b}^{2 x+1}(\mathbb{G}(1,3))$.

Proof. Let $Q \subset \mathbb{P}^{3}$ be a smooth quadric hypersurface. The Fano variety of lines $F_{1}(Q)$ is the zero locus of a section of the following bundle,

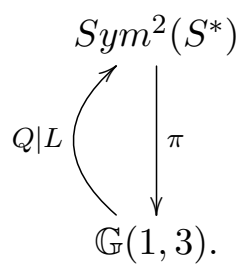


A smooth conic in $\mathbb{P}^{5}$ determines uniquely a 2-plane, thus in the Plücker embedding $\mathbb{G}(1,3) \subset$ $\mathbb{P}^{5}$, we have that

(1) $F_{1}(Q)$ determines two 2-planes if $\operatorname{rank}(Q)$ is either 4 or 2 ,

(2) $F_{1}(Q)$ determines a single 2-plane if $\operatorname{rank}(Q)$ is either 1 or 3.

Exchanging such planes gives rise to a $\mathbb{Z} / 2$-action on $\mathbb{G}(2,5)$, the Grassmannian of 2-planes in $\mathbb{P}^{5}$. Such a $\mathbb{Z} / 2$-action on $\mathbb{G}(2,5)$ preserves the Orthogonal Grassmannian $\mathbb{O} \mathbb{G}$, hence inducing a $\mathbb{Z} / 2$-action on the blowup $\mathbf{H i l b}^{2 x+1}(\mathbb{G}(1,3))$.

Observe that there is a $S L_{4} \mathbb{C}$-action on Hilb induced by the action of $S L_{4}$ on $\mathbb{P}^{3}$. This action stratifies Hilb in $S L_{4}$-orbits compatible with the divisors $E_{1,1}^{+}, E_{2}^{+}$. Notice that $\mathbb{Z} / 2$ acts trivially (as the identity) over $S L_{4}$-orbits of codimension 2 . In codimension 1 , we have that $\mathbb{Z} / 2$ acts as the identity on the exceptional divisors $E_{2}^{+}$and $E_{1,1}^{+}$.

Definition 3.6. Considering the $\mathbb{Z} / 2$-action defined above, let us denote the quotient space $X^{+}:=\mathbf{H i l b} /(\mathbb{Z} / 2)$

Let $\bar{M}=\bar{M}_{0,0}(\mathbb{G}, 2)$ be the Kontsevich moduli space of degree 2 stable maps into the Grassmannian $\mathbb{G}=\mathbb{G}(1,3)$.

Lemma. There is a nontrivial globally defined $\mathbb{Z} / 2$-action on the Kontsevich moduli space $\bar{M}_{0,0}(\mathbb{G}(1,3), 2)$. 
Proof. Observe we have a generic 2-1 morphism from the Kontsevich moduli space $\bar{M}=$ $\bar{M}_{0,0}(\mathbb{G}(1,3), 2)=\left\{\left(C, C^{*}\right\}\right.$ to the space $X$ of complete quadric surfaces defined by

$$
\left(C, C^{*}\right) \mapsto\left(\bigcup_{L \in C} L, C^{*}\right)
$$

where $\left(S, C^{*}\right)$, following the notation of the proposition 2.1, stands for a surface $S$ and the curve $C^{*}$ as the marking. This map is 2 to 1 over the open subset parametrizing smooth quadric surfaces as well as over the divisor of complete quadrics of rank 2. Indeed, if $\bigcup_{L \in C} L$ sweeps out a smooth quadric $S$, then $L$ is a ruling of $S$. The other ruling induces another conic $C^{\prime}$ which gets mapped to $S$. The situation is similar over the locus of complete quadrics of rank 2. Notice that this map is $1-1$ outside such two regions. We now define the $\mathbb{Z} / 2$-action on $\bar{M}$ by identifying the two curves $C$ and $C^{\prime}$.

Corollary. The quotient of $\bar{M}_{0,0}(\mathbb{G}, 2)$ by the $\mathbb{Z} / 2$-action is isomorphic to $X$. In particular, the quotient is smooth.

Proof. Let $Z$ denote the quotient of $\bar{M}$ by the $\mathbb{Z} / 2$-action defined above. Observe that $X$ and $Z$ are birational and there is a bijection between them. Zariski Main Theorem implies the corollary.

\subsection{Proof of Theorem B}


The movable cone $\operatorname{Mov}(X)$ is the closed cone bounded by $H_{1}, H_{2}, H_{3}$ and $P$. In this section we describe all the models $X(D)$, where $D \in \operatorname{Mov}(X)$. Moreover, we interpret the spaces constructed thus far as $X(D)$ for some $D$ in $\operatorname{Mov}(X)$

Proposition. There is a morphism from $X^{+}:=\mathbf{H i l b} /(\mathbb{Z} / 2)$ to the $\mathbb{Z} / 2$-Chow variety defined by forgetting the scheme structure and only remembering its cycle class. Likewise, there is morphism from the space of complete quadrics $X$ to the same $\mathbb{Z} / 2$-Chow variety.

Proof. Consider the following spaces. Let $I=\left\{\left(C, C^{*}, \Lambda\right)\right\}$ be the incidence correspondence such that $C$ is a connected, arithmetic genus zero, degree two curve in $\mathbb{G}(1,3) \cap \Lambda$ and $C^{*}$ is the dual curve in $\Lambda$. Let $\bar{M}_{0,0}(\mathbb{G}, 2)$ be the Kontsevich space of degree two stable maps into the Grassmannian $\mathbb{G}=\mathbb{G}(1,3)$. Let $\mathcal{C}$ denote the Chow variety of conics in $\mathbb{P}^{5}$ which are contained in $\mathbb{G}(1,3)$. The incidence correspondence $I$ admits a map to both $\bar{M}_{0,0}(\mathbb{G}, 2)$ and Hilb by projection to the first two factors, and by projection to the first and third factors. By projection to the first factor, we get a map to $\mathcal{C}$. Since the morphisms $K h$ and $C h$ are small 
contractions, and $\mathbb{Z} / 2$ acts trivially in $S L_{4}$-orbits of codimension 2 and higher, then the Chow variety $\mathcal{C}$ inherits a $\mathbb{Z} / 2$-action. We thus have the following,

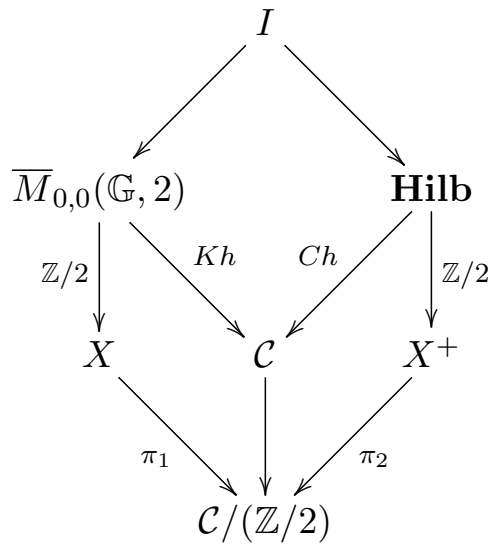

The existence of morphisms $\pi_{1}$ and $\pi_{2}$ follows from the fact that $X$ as well as $X^{+}$are $\mathbb{Z} / 2$ quotients.

In the main theorem of this section we list only the models $X(D)$ for which we have found a moduli interpretation.

Theorem B. Let $D$ be an effective divisor in the space of complete quadric surfaces $X$ and let

$$
X(D)=\operatorname{Proj}\left(\bigoplus_{m \geq 0} H^{0}(X, m D)\right)
$$

be the model induced by $D$. Then, we have the following models for $X(D)$,

1. $X(D) \cong X$ for $D$ contained in the cone bounded by $H_{1}, H_{2}$ and $H_{3}$.

2. $X\left(H_{1}\right) \cong \mathbf{H i l b}^{(x+1)^{2}}\left(\mathbb{P}^{3}\right) \cong \mathbb{P}^{9}$ and $f: X \rightarrow X\left(H_{1}\right)$ contracts the divisors $E_{1}$ and $E_{2}$. 
3. $X\left(H_{3}\right) \cong \mathbf{H i l b}^{(x+1)^{2}}\left(\mathbb{P}^{3 *}\right) \cong \mathbb{P}^{9 *}$ and $g: X \rightarrow X\left(H_{3}\right)$ contracts the divisors $E_{3}$ and $E_{2}$.

4. $X\left(H_{2}\right) \cong \mathbf{C h o w}_{2}(1, X)$ and $\phi: X \rightarrow X\left(H_{2}\right)$ contracts the divisor $E_{2}$.

5. $X(D) \cong \mathcal{C} /(\mathbb{Z} / 2)$ where $\mathcal{C}$ is the Chow variety of Proposition 21 and $D=t H_{1}+(1-t) H_{3}$ for $0<t<1$. The map $\phi_{1}: X \rightarrow \mathcal{C} /(\mathbb{Z} / 2)$ is a small contraction where the intersection $E_{1} \cap E_{3}$ gets contracted.

6. $X(D) \cong X^{+}$for $D$ contained in the domain bounded by $H_{1}, H_{3}$ and $P$. The map $\phi_{2}$ : $X^{+} \rightarrow \mathcal{C} /(\mathbb{Z} / 2)$ is the flip of $\phi_{1}$, where the flipping locus consists of subschemes supported on a line.

7. $X(P) \cong \mathbb{G}(2,5) /(\mathbb{Z} / 2)$ for $P$ as defined in page 8 .

The result above can be best seen from the following diagram:

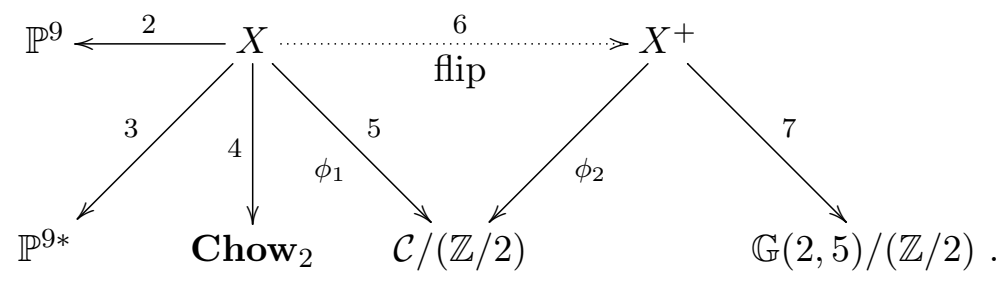

where $\phi_{1}$ and $\phi_{2}$ are small contractions and the other maps, except for the flip, are all divisorial contractions. Observe that from corollary 3.4 and proposition 3.2 we know abstractly all the divisorial contractions of $X$ or $X^{+}$induced by $\operatorname{Mov}(X)$. In Theorem B, we list only those models for which we are exhibiting a moduli structure.

Proof of Theorem B. (1), (2), (3) follow from Theorem A, which is the description of the nef cone $\operatorname{Nef}(X)$, as well as from Vainsencher's Theorem. 
(4) This is established by the corollary 3.4 in section 3.4 .

(5) Let $C_{1,2}$ be the curve defined in table on page 9 , and whose deformations cover the codimension 2 subvariety $E_{1} \cap E_{3}$. Observe that for any divisor $D=t H_{1}+(1-t) H_{3}$ where $0<t<1$, we have that $C_{1,2} \cdot D=0$, which says that $\phi_{D}$ contracts the codimension 2 locus $E_{1} \cap E_{3}$. The locus which is contracted does not get any larger as the map $X \rightarrow \mathcal{C} /(\mathbb{Z} / 2)$ behaves locally similar to that of the diagram (Equation 3.7) and by the observation made about the $\mathbb{Z} / 2$-action on $S L_{4}$-orbits, its exceptional locus behaves as in (14).

(6) By definition, the morphism $\phi_{2}: X^{+} \rightarrow \mathcal{C} /(\mathbb{Z} / 2)$ is the flip of $\phi_{1}: X \rightarrow \mathcal{C} /(\mathbb{Z} / 2)$, if for any divisor $D$ in the domain bounded by $H_{1}, H_{3}$ and $P$, then both $-D$ is $\phi_{1}$-ample and $D$ is $\phi_{2}$-ample.

We remark that the $\mathbb{Z} / 2$-action on $X^{+}$behaves like the identity over the locus which is flipped. Hence in the following analysis we can neglect the $\mathbb{Z} / 2$-action altogether.

We verify that any $D$ as above is $\phi_{2}$-ample. Note that $\phi_{2}^{-1}(p) \cong \mathbb{P}^{1}$. Indeed, for $L \subset \mathbb{G}(1,3)$, where $L$ denotes a line, consider the tangent space $\mathbb{T}_{L} \mathbb{G}(1,3) \cong \mathbb{P}^{3}$. Now $\mathbb{T}_{L} \mathbb{G}(1,3) \cap \mathbb{G}(1,3)$ is a quadric of rank 1 (a double plane) with a double line $2 L$ on it. The pencil of planes containing $L$ are different points of Hilb, however they all map to the same point $[2 L]$ of the Chow variety $\mathcal{C}$. This means that the fiber of $\phi_{2}$ over the point $[2 L]$ is a pencil of planes, hence $\mathbb{P}^{1}$. Now let $D=a \bar{H}_{1}+b \bar{H}_{3}+c P$ for positive $a, b, c \in \mathbb{Q}$ and where $\bar{H}_{1}$ and $\bar{H}_{3}$ are defined in Pic(Hilb) as follows. $\bar{H}_{1}=\left\{(C, \Lambda) \mid C \cap \sigma_{2}(P l) \neq \emptyset\right\}$ for a fixed plane $P l \subset \mathbb{P}^{3}$, 
and $\bar{H}_{3}=\left\{(C, \Lambda) \mid C \cap \sigma_{1,1}(p) \neq \emptyset\right\}$ for a fixed point $p \in \mathbb{P}^{3}$. Consequently, for the curve $\gamma=\phi_{2}^{-1}(2 L)$, we have

$$
\begin{aligned}
\gamma \cdot D & =\gamma \cdot\left(a \bar{H}_{1}+b \bar{H}_{3}+c P\right) \\
& =c(\gamma \cdot P) \\
& =c\left(\gamma \cdot \sigma_{1}\right) \quad \text { in } \mathbb{G}(2,5) \\
& >0 .
\end{aligned}
$$

Thus, $D$ is $\phi_{2}$-ample.

Let us now describe the fiber $\phi_{1}^{-1}(p)$. By Nakai's ampleness criterion $-D$ will be $\phi_{1}$-ample if and only if $D \cdot \gamma<0$ for any curve $\gamma$ contained in the fiber of $\phi_{1}$. The curve $C_{1,2}$ is contained in such a fiber as it is contracted by $\phi_{1}$. Hence,

$$
\begin{aligned}
C_{1,2} \cdot D & =C_{1,2} \cdot\left(a H_{1}+b H_{3}+c P\right) \\
& =c\left(C_{1,2} \cdot P\right) \\
& =-c\left(C_{1,2} \cdot H_{2}\right) \\
& <0 .
\end{aligned}
$$

Thus $-D$ is $\phi_{1}$-ample.

(7) Follows by construction. This completes the proof of Theorem B. 


\section{CHAPTER 4}

\section{PREVIOUS AND FUTURE WORK}

Let us comment on the relation of Semple's work to the results in this thesis. Additionally, let us elaborate on the relation of our work to GIT theory and the theory of wonderful varieties.

Consider the space of complete quadric surfaces $X \subset \mathbb{P}^{9} \times \mathbb{P}^{20} \times \mathbb{P}^{9 *}=\mathbb{P}_{1} \times \mathbb{P}_{2} \times \mathbb{P}_{3}$. Theorem B says that the image of the projection map $\rho_{i}: X \rightarrow \mathbb{P}_{i}$ is isomorphic to $X\left(H_{i}\right)$, for $1 \leq i \leq 3$. One can also consider the projection

$$
\rho_{i, j}: X \rightarrow \mathbb{P}_{i} \times \mathbb{P}_{j}
$$

for $1 \leq i<j \leq 3$. Semple focused on the projections $\rho_{2}$ and $\rho_{1,3}$. For example, he denotes the space $\rho_{2}(X)$ by $C_{9}^{92}[19]$ and carefully studies its singularities. By proposition 3.2, the projection $\rho_{1,2}(X)$ (respectively, $\rho_{2,3}(X)$ ) is a divisorial contraction isomorphic to $X_{1}$ (respectively, $Y_{1}$ ). The projection $\rho_{1,3}(X)$ is of a different kind. It is a small contraction which Semple denotes by $W_{9}$. He carefully analyzes the singularities of $W_{9}$ as well as its geometry. Thus far, we may categorize the study of these spaces as analyzing the models arising from divisors in the nef cone of $X$.

Birational models of $X$ not analyzed in (2) arise when we study models $X(D)$ induced by divisors $D$ which are not nef, but which are contained in the movable cone. One such example is the flip of $X$ over $\rho_{1,3}(X)$. 
On the other hand, the space of complete $(n-1)$-quadrics can be obtained as a GIT quotient. Indeed, De Concini and Procesi in (5) constructed the "wonderful compactification" of a symmetric variety. Viewing $S L_{n+1} \mathbb{C} \cong S L_{n+1} \mathbb{C} \times S L_{n+1} \mathbb{C} / \Delta$ as a symmetric variety, one can consider the wonderful compactification $\bar{G}=\overline{S L_{n+1} \mathbb{C}}$. This is an $H$-variety, where $H$ is the fixed subgroup of the $S L_{n+1}$-involution which permutes the factors. Thus, we can take the GIT quotient $\bar{G}^{s s} / / H$. This quotient is a compactification of $S L_{n+1} / H$ which, for a suitable choice of linearization of $H$, is isomorphic to complete $(n-1)$-quadrics (19). This point of view suggests that we might understand Theorem B and C as a variation of GIT. We would like to comeback to this point in the future.

In the same vein, Theorem B has an interpretation in terms of representation theory and the theory of wonderful varieties. Indeed, the family of smooth quadric hypersurfaces in $\mathbb{P}^{n}$, which we denote by $X^{\circ}$, has the structure of a symmetric variety. That is to say, $X^{\circ}=$ $S L_{n+1} / \tilde{S O}(n+1)$, where $\tilde{S O}(n+1)$ is the fixed subgroup of the involution $\sigma(A)={ }^{t} A^{-1}$. We get the wonderful compactification of $X^{\circ}$ by looking at representations of $S L_{n+1}$ such that there is a vector $v$ whose stabilizer is precisely $\tilde{S O}(n+1)$. De Concini and Procesi $(5)$ showed in a great deal of generality that such a representation of $S L_{n+1}$ exists and that it coincides with the space of complete $(n-1)$-quadrics. Then, it is natural to ask: what kind of representations of $S L_{n+1} \mathbb{C}$ give rise to the birational models $X(D)$ ? In particular, which gives rise to the flip of $X$. Ideas contained in (5) realize the models $X(D)$, where $D \in \operatorname{Nef}(X)$, as compactifications arising from representations of $S L_{4} \mathbb{C}$, it may happen that all the models $X(D)$ arise this way. 
For example, the space of complete 2-quadrics is a wonderful compactification $X=\overline{S L_{4} / \tilde{S O}(4)}$. The flip $X^{+}$, as defined in 3.6, has two closed $S L_{4}$-orbits. This implies that $X^{+}$is not a wonderful variety, for such varieties have a unique closed orbit. Observe that $\mathfrak{s l}_{4}=\mathfrak{s o}(6)$ are isomorphic as Lie algebras. Then, we may try to investigate irreducible representations of $S O(6)$ with suitable invariants in order to find $X^{+}$. We should keep in mind that $S O(6)$ is not a Lie group of adjoint type and consequently the theory of wonderful varieties as in (5) does not apply. That is to say, there is no wonderful compactification for $S O(6)$ or quotients of it. Nonetheless, the "log homogeneous compactification" of $\overline{S O}(6)$ introduced by M. Brion (20) is expected to have two closed orbits (21), which suggests that $X^{+}$may be closed related to a certain compactification $\overline{S O(6) / H}$ for a suitable subgroup $H$. Since numerous flips of the wonderful variety $X=\overline{S L_{n+1} / \tilde{S O}(n+1)}$ are expected to exist, the previous example suggests there exist certain representations of groups isogenous to $S L_{n+1}$ which are not considered by the theory of wonderful varieties, but that nonetheless give rise to flips of wonderful varieties. We would like to pursue these ideas in the future. 


\section{CITED LITERATURE}

1. Semple, J. G.: On complete quadrics I. J. Lon. Math. Soc, pages 258-267, 1948.

2. Semple, J. G.: On complete quadrics II. J. Lon. Math. Soc, pages 280-287, 1952.

3. Schubert, H.: Kalkul der abzahlenden geomretrie. Leipsig, (Reprinted by Springer-Verlag, Berlin (1979)), 1879.

4. Kleiman, S. and Thorup, A.: Intersection theory and enumerative geometry: a decade in review. AMS Proc. of Symp. Pure Math (Algebraic geometry: Bowdoin 1985), 46$2: 321-370,1987$.

5. De Concini, C. and Procesi, C.: Complete symmetric varieties. Invariant Theory, 1980. Springer-Verlag.

6. Lazarsfeld, R.: Positivity in Algebraic Geometry I, classical setting: line bundles and linear series. Springer-Verlag, 2004.

7. Birkar, P., Cascini, P., Hacon, C., and McKernan, J.: Existence of minimal models for varieties of log general type. J. Amer. Math Soc., (2):405-468, 2010.

8. Hu, Y. and Keel, S.: Mori dream spaces and GIT. Michigan Mathematical Journal, 48(6):331-348, 2000. Dedicated to William Fulton on the ocasion of his 60th birthday.

9. Ein, L., Lazarsfeld, M., Mustaţă, M., Nakamaye, M., and Popa, M.: Asymptotic invariants of base loci. Ann. Inst. Fourier, 56(6):1701-1734, 2006.

10. Ein, L., Lazarsfeld, M., Mustaţă, M., Nakamaye, M., and Popa, M.: Restricted volumes and base loci of linear series. Amer. J. Math, 131(3):607-651, 2009.

11. Hassett, B.: Classical and minimal models of the moduli space of curves of genus two. Geometric methods in algebra and number theory, 235:169-192, 2005. Birkhuser, Boston, MA,. 
12. Hassett, B. and Hyeon, D.: Log minimal models for the moduli space of curves: The first flip. Ann. of Math., 177(3):911-968, 2013.

13. Hassett, B. and Hyeon, D.: Log canonical models for the moduli space of curves: The first divisorial contraction. Transations of the AMS, 361(4):1065-1080, 2009.

14. Chen, D. and Coskun, I.: Stable base locus of Kontsevich moduli spaces. Michigan Mathematical Journal, 59(2):435-466, 2010.

15. Vainsencher, I.: Schubert calculus for complete quadrics. In Enumerative geomety and classical algebraic Geometry, pages 199-235. Boston, Mass, Birkhäuser, 1982.

16. Thorup, A.: Parameter spaces for quadrics. Banach Center Publications, 36, 1996.

17. Tyrell, J.: Complete quadrics and collineations in $S_{n}$. Mathematic, 3:69-79, 1956.

18. Eisenbud, D.: Commutative Algebra with a view towards Algebraic Geometry. In Grad. Texts Math.. Springer-Verlag, 1995.

19. Kanna, S. S.: Remark on the wonderful compactification of semisimple algebraic groups. Proc. Indian Acad. Sci., 109(3):241-256, 1999.

20. Brion, M.: Log homogeneous varieties. In In Proccedings of the XVIth Latin American Algebra Colloquium (Spanish), pages 1-39. Madrid, Rev. Mat. Iberoamericana, 2007.

21. Huruguen, M.: Log homogeneous compactifications for some classical groups. preprint.

22. Harris, J. and Tu, L.: On symmetryc and skew-symmetric determinantal varieties. Topology, 23:71-84, 1984.

23. De Fernex, T., Ein, L., and M., M.: Shokurov's ACC conjecture for log canonical thresholds on smooth varieties. Duke Math., 152:93-114, 2010.

24. De Fernex, T., Ein, L., and Mustata, M.: Bounds for log canonical thresholds with applications to birational rigidity. Math. Res. Lett., 10:219-236, 2003.

25. Kovács, S.: The cone of curves of a $k 3$ surface. Math. Ann, 300(4):681-691, 1994. 
26. Thaddeus, M.: Complete collineations revisited. arXiv:math/9808114, 2001.

27. Chen, D.: Mori's program for the Kontsevich moduli space $\bar{M}_{0,0}\left(\mathbb{P}^{3}, 3\right)$. Int. Math. Res. Not., 235:17 pp., 2005.

28. Coskun, I., Harris, J., and Star, J.: Effective cone of the Kontsevich moduli space. Int. Math. Res. Not., 235:17 pp., 2005.

29. Grothendieck, A.: Les schémas de hilbert. In Séminare Bourbaki, page exposé. Paris, IHP, 1961.

30. Chasles, M.: Dètermination du nombre de sections coniques qui doivent toucher cinq courbes donneés d'ordre quelconque, ou satisfaire à diverses autres conditions. C.R. Acad. Sci. Paris, 58:222-226, 1864.

31. Arcala, D., Bertram, A., Coskun, I., and Huizenga, J.: The minimal model program for the Hilbert scheme of points on the plane and Bridgeland stability. Advances in Mathematics, 325:580-626, 2013.

32. De Concini, C., Goresky, M., MacPherson, R., and Procesi, C.: On the geometry of quadrics and their degenerations. Comment. Math. Helvetici, 63:337-413, 1988.

33. Severi, F.: Riflessioni intorno ai problemi numerative... Rend. del R. Est. Lomb. di Scienze e Letter, 54:243-254, 1921.

34. Bialynicki-Birula: Some theorems on actions of algebraic groups. Ann. of Math., (98):480497, 1973.

35. Debarre, O., Ein, L., Lazarsfeld, R., and Voisin, C.: Pseudoeffective and nef classes on abelian varieties. Compos. Math., 147:1793-1818, 2011.

36. Voisin, C.: Coniveau 2 complete intersection and effective cones. Geo. Funct. Anal., 19:1494-1513, 2010.

37. Peternell, T.: Submanifolds with ample normal bundle and conjecture of Hartshorne. Comtemp. Math., 496:317-330, 2009.

38. Laksov, D.: complete quadrics and linear maps. AMS Proc. of Symp. Pure Math (Algebraic geometry: Bowdoin 1985), 46-2:371-387, 1987. 
39. Kollár, J.: The structure of algebraic threefolds: an introduction to Mori's Program. Bulletin of the Amer. Math. Soc., 17(2):211-273, 1987.

40. Hartshorne, R.: Algebraic Geometry. In Graduate Texts in Mathematics. Springer-Verlag, 1978.

41. Griffiths, P. and Harris, J.: Principles of Algebraic Geometry. Wiley Interscience, 1978.

42. Kollár, J. and Mori, S.: Birational geometry of algebraic varieties. Cambridge Traces in Mathematics., 2008.

43. Hartshorne, R.: Deformation Theory. In Graduate Texts in Mathematics.. SpringerVerlag, 2009. 


\section{César Lozano Huerta}

University of Illinois at Chicago

Department of Mathematics, Statistics, and Computer Science 322 Science and Engineering Offices (M/C 249)

851 S. Morgan Street

Chicago, IL 60607-7045. USA

Citizenship: Mexican
Email: lozano@math.uic.edu

Homepage: http://www.math.uic.edu/ lozano

\section{Education}

4th year Ph.D student, The University of Illinois at Chicago, 2010-

Advisor: Izzet Coşkun.

M.S. Mathematics, University of Utah, 2008-2010.

Aaron Bertram, University of Utah, (Advisor)

M.S. Mathematics, CINVESTAV-IPN, México 2008.

Dissertation: From Quantum Cohomology to Integrable Systems.

Ernesto Lupercio, Cinvestav-IPN, (Advisor)

B.S. Physics and Mathematics, Instituto Politécnico Nacional, México 2006.

\section{Research}

I am an algebraic geometer who specializes in birational geometry and the theory of moduli spaces, with a view towards applications in enumerative geometry, representation theory and combinatorics. My research focuses on using techniques from the Minimal Model Program in order to understand different compactifications of symmetric spaces such as Lie Groups and quotients of them.

\section{Publications}

Birational Geometry of Complete Quadrics. preprint

\section{Fellowships}

Research Assistantship, under Prof I. Coskun. University Of Illinois at Chicago, Fall 2013.

Research Assistantship, under Prof I. Coskun. University Of Illinois at Chicago, Fall 2012.

Research Assistantship, under Prof I. Coskun. University Of Illinois at Chicago, Spring 2012.

Teaching Assistantship, University Of Illinois at Chicago, 2010- .

National Fellowship to conduct PhD studies abroad. CONACyT, México , 2008-2010 . 
National Fellowship to study a semester abroad. CONACyT, México 2007.

National Fellowship to conduct M.A. studies. CONACyT, México, 2006-2008.

\section{Invited Seminar Talks}

Harvard University, Harvard/MIT Algebraic Geometry Seminar, April. 2014.

Johns Hopkins University, Algebraic Geometry and Number Theory Seminar, Feb. 2014.

Rice University, Algebraic Geometry Seminar, Jan. 2014.

University of Chicago, Farb and Friends Seminar, Feb. 2012.

University of Illinois at Chicago, Math Circle Sep. 2011.

\section{Invited Colloquia}

Northern Illinois University, Math Department Colloquium, Feb. 2014.

University of Illinois at Chicago, Graduate Student Colloquium, Sep. 2013.

University of Illinois at Chicago, Graduate Student Colloquium, Sep. 2012.

University of Illinois at Chicago, Graduate Student Colloquium, Feb. 2012.

\section{Conference Talks and Poster Sessions}

Midwest Algebraic Geometry Graduate Conference, Feb. 2014

Birational geometry of complete quadrics. AGNES, Boston College. Fall 2013.

\section{SERVICE}

Co-organizer of the UIC-Graduate Student Colloquium, Fall 2012.

Founder and Co-organizer of the UIC-Graduate Student Colloquium, Spring 2012.

Organizer of the UIC-Graduate Algebraic Geometry Seminar, Fall, 2011. 


\section{Conferences and Workshops Attended}

Compactifyin Moduli Spaces, March 27-31, 2013. CRM, Spain.

A celebration of Algebraic Geometry,(Joe Harris' 60th birthday) August 25-28, 2011. Harvard University, USA.

Summer School on Riemann surfaces, July 02-22, 2011. Park City, USA.

School on Moduli Spaces, January 04-14, 2011. Cambridge University, United Kingdom.

School on Birational Geometry and Moduli spaces, June 01-11, 2010. Salt Lake City, USA.

Recent Progress on Moduli Space of Curves, March 16-21, 2008. Banff, Canada.

Topological Methods in Quantum Field Theory, July 2-21, 2008. Villa de Leyva, Colombia.

\section{Teaching Experience}

MATH 180 Calculus II, Spring 2013. UIC.

MATH 180 Calculus I, Summer 2012. UIC.

MATH 179-ESP Calculus I, Fall 2012. UIC.

MATH 180 Calculus I, Spring 2011. UIC.

MATH 181 Calculus II, Fall 2010. UIC.

\section{Miscellaneous}

University Of Illinois at Chicago, Department of Mathematics

Visiting student, Spring 2010.

University Of Michigan, Department of Mathematics

Visiting student, Fall 2007.

LANGUAGES

Spanish: Native.

English: Fluent.

French: Medium.

Computer Skills

C, C++, Linux, html, $\mathrm{LAT}_{\mathrm{E}} \mathrm{X}$. 


\section{References}

Izzet Coşkun

Professor of Mathematics

University of Illinois at Chicago

coskun@math.uic.edu

Renzo Cavalieri

Assistant Professor

Colorado State University

renzo@math.colostate.edu

Dawei Chen

Assistant Professor

Boston College

chen@bc.edu

Lawrence Ein

Professor of Mathematics

University of Illinois at Chicago

ein@math.uic.edu

Iddo Samet (teaching)

Research Assistant Professor

University of Illinois at Chicago

samet@math.uic.edu 